\title{
NUMERICAL CHARACTERIZATION OF SOME TORIC FIBER BUNDLES
}

\author{
STÉPHANE DRUEL AND FEDERICO LO BIANCO
}

\begin{abstract}
Given a complex projective manifold $X$ and a divisor $D$ with normal crossings, we say that the logarithmic tangent bundle $T_{X}(-\log D)$ is R-flat if its pull-back to the normalization of any rational curve contained in $X$ is the trivial vector bundle. If moreover $-\left(K_{X}+D\right)$ is nef, then the $\log$ canonical divisor $K_{X}+D$ is torsion and the maximally rationally chain connected fibration turns out to be a smooth locally trivial fibration with typical fiber $F$ being a toric variety with boundary divisor $D_{\mid F}$.
\end{abstract}

\section{CONTEnTs}

1. Introduction

2. Notation, convention and used facts

3. A canonical bundle formula for generically isotrivial fibrations

4. Descent of vector bundles

5. Preparation for the proof of the main results

6. Proofs

References

\section{INTRODUCTION}

Let $X$ be a smooth projective algebraic variety $X$ over the field of complex numbers and let $D$ be a divisor with normal crossings. The structure of pairs $(X, D)$ with trivial logarithmic tangent bundle $T_{X}(-\log D)$ is well understood by a result of Winkelmann (see [Win04]). They are called semiabelic varieties. The simplest examples of pairs $(X, D)$ with trivial logarithmic tangent bundle $T_{X}(-\log D)$ are pairs $(A, 0)$ where $A$ is an abelian variety, and pairs $(X, D)$ where $X$ is a smooth toric variety with boundary divisor $D$. If $X$ is smooth projective semiabelic variety, then the algebraic group $G:=\operatorname{Aut}^{0}(X, D)$ is a semiabelian group which acts on $X$ with finitely many orbits. Moreover, the $G$-orbits in $X$ are exactly the strata defined by $D$. As a consequence, the albanese map is a smooth locally trivial fibration with typical fiber $F$ being a toric variety with boundary divisor $D_{\mid F}$. In particular, semiabelic varieties are toric fiber bundles over abelian varieties.

However, from the point of view of birational classification of algebraic varieties, it is more natural to consider the case where the logarithmic tangent bundle $T_{X}(-\log D)$ is numerically flat (see Definition 2.11 for this notion). If $D=0$, then $X$ is covered by an abelian variety, as a classical consequence of Yau's theorem on the existence of a Kähler-Einstein metric. In the present paper, we obtain a numerical characterization of a class of toric fiber bundles containing pairs $(X, D)$ with numerically flat logarithmic tangent bundle $T_{X}(-\log D)$ (see Corollary 1.7).

Our main result is the following. A vector bundle $\mathscr{E}$ on a projective variety $X$ is called R-flat if $\nu^{*} \mathscr{E}$ is the trivial vector bundle for any morphism $\nu: \mathbb{P}^{1} \rightarrow X$ (see paragraph 2.10).

Theorem 1.1. Let $(X, D)$ be a log smooth reduced pair with $X$ projective. Suppose that $-\left(K_{X}+D\right)$ is nef and that $T_{X}(-\log D)$ is $R$-flat. Then there exist a smooth projective variety $T$ with $K_{T} \equiv 0$ as well as a smooth morphism with connected fibers $a: X \rightarrow T$. The fibration $(X, D) \rightarrow T$ is locally trivial for the analytic topology and any fiber $F$ of the map $a$ is a smooth toric variety with boundary divisor $D_{\mid F}$. Moreover, $T$ contains no rational curve.

In fact, a more general statement is true (see Theorem 6.1) but its formulation is somewhat involved.

Remark 1.2. Let $(X, D) \rightarrow T$ is a toric fiber bundle over a projective manifold $T$ with $K_{T} \equiv 0$. Suppose in addition that $T$ contains no rational curve. Then $T_{X}(-\log D)$ is obviously R-flat. Moreover, $K_{X}+D \equiv 0$ by Theorem 3.3. 
Remark 1.3. In the setup of Theorem 1.1, we expect that $T$ is a torus quotient. Indeed, by the BeauvilleBogomolov decomposition theorem, $T$ admits a finite étale cover that decomposes into the product of an abelian variety and a simply-connected Calabi-Yau manifold. On the other hand, a folklore conjecture asserts that any projective Calabi-Yau manifold contains a rational curve.

This motivates the following question.

Question 1.4. Let $(X, D)$ be a log smooth reduced pair with $X$ projective. Suppose that $-\left(K_{X}+D\right)$ is nef and that $T_{X}(-\log D)$ is $R$-flat. Is $X$ a toric fiber bundle over an étale quotient of an abelian variety?

The following results are rather easy consequences of Theorem 1.1 above.

Corollary 1.5. Let $(X, D)$ be a log smooth reduced pair with $X$ projective. Suppose that $-\left(K_{X}+D\right)$ is nef and that $T_{X}(-\log D)$ is $R$-flat. Suppose in addition that $X$ is simply-connected and that $h^{p, 0}(X)=0$ for all $1 \leqslant p \leqslant \operatorname{dim} X$. Then $X$ is a smooth toric variety with boundary divisor $D$.

The next question is a special case of Question 1.4 above.

Question 1.6. Let $(X, D)$ be a log smooth reduced pair with $X$ projective. Suppose that $-\left(K_{X}+D\right)$ is nef and that $T_{X}(-\log D)$ is $R$-flat. Suppose in addition that $X$ is simply-connected. Is $X$ a smooth toric variety with boundary divisor $D$ ?

Corollary 1.7. Let $(X, D)$ be a log smooth reduced pair with $X$ projective. Suppose that $T_{X}(-\log D)$ is numerically flat. Then there is a smooth morphism $a: X \rightarrow T$ with connected fibers onto a torus quotient $T$. The fibration $(X, D) \rightarrow T$ is locally trivial for the analytic topology and any fiber $F$ of the map a is a smooth toric variety with boundary divisor $D_{\mid F}$.

The next result says in particular that Corollary 1.7 applies to pairs with flat logarithmic tangent sheaf.

Proposition 1.8. Let $(X, D)$ be a log smooth reduced pair with $X$ projective. If $T_{X}(-\log D)$ admits a holomorphic connection, then it is numerically flat.

The proof of Theorem 1.1 relies in part on a descent theorem for vector bundles which is of independent interest. Our result extends [BdS09, Theorem 1.1] to the relative setting.

Theorem 1.9. Let $f: X \rightarrow Y$ be a projective morphism with connected fibers of normal, quasi-projective varieties. Suppose that $X$ is smooth and that $Y$ is klt. Suppose in addition that $f$ has rationally chain connected fibers. Let $\mathscr{E}$ be a locally free, $f$-relatively $R$-flat sheaf on $X$. Then there exists a locally free sheaf $\mathscr{G}$ on $Y$ such that $\mathscr{E} \cong f * \mathscr{G}$.

Structure of the paper. Section 2 gathers notation, global conventions, and known results that will be used throughout the paper. We also establish some facts. In particular, we establish a number of properties of R-flat vector bundles. In section 3, we prove a canonical bundle formula for generically isotrivial fibrations. Section 4 is devoted to the proof of Theorem 1.9. Section 5 prepares for the proof of the main results. With these preparations at hand, the proof of Theorem 1.1 as well as the proofs of Corollaries 1.5 and 1.7 and Proposition 1.8 , which we give in Section 6 , become reasonably short.

Acknowledgements. The authors would like to thank Enrica Floris for discussions concerning the canonical bundle formula. We would also like to thank Benoit Claudon for helpful conversations.

The first author was partially supported by the ERC project ALKAGE (ERC grant Nr 670846), the CAPESCOFECUB project Ma932/19 and the ANR project Foliage (ANR grant Nr ANR-16-CE40-0008-01).

The second author was supported by the LABEX MILYON (ANR-10-LABX-0070) of Université de Lyon, within the program Investissements d'Avenir (ANR-11-IDEX-0007) operated by the ANR.

\section{Notation, CONVEntion And USED FACtS}

2.1. Global conventions. Throughout the paper, all varieties are assumed to be defined over the field of complex numbers. Given a variety $X$, we denote by $X_{\text {reg }}$ its smooth locus.

2.2. Pull-back of Weil divisors. Let $\psi: X \rightarrow Y$ be a dominant equidimensional morphism of normal varieties, and let $D$ be a Weil $\mathbb{Q}$-divisor on $Y$. The pull-back $\psi^{*} D$ of $D$ is defined as follows. We define $\psi^{*} D$ to be the unique $\mathbb{Q}$-divisor on $X$ whose restriction to $\psi^{-1}\left(Y_{\text {reg }}\right)$ is $\left(\psi_{\mid \psi^{-1}\left(Y_{\text {reg }}\right)}\right)^{*}\left(D_{\mid Y_{\text {reg }}}\right)$. This construction agrees with the usual pull-back if $D$ is $\mathbb{Q}$-Cartier. 
2.3. Exceptional divisor. We will need the following definition.

Definition 2.1. Let $f: X \rightarrow Y$ be a dominant rational map of normal varieties. Suppose in addition that $Y$ is projective. A prime divisor $Q$ on $X$ is called $f$-exceptional if $\operatorname{codim}_{Y} f(Q) \geqslant 2$.

Remark 2.2. Setup as in Definition 2.1. The image $f(Q)$ of $Q$ is well-defined since $Y$ is projective by assumption.

2.4. Projective space bundle. If $\mathscr{E}$ is a locally free sheaf of finite rank on a variety $X$, we denote by $\mathbb{P}_{X}(\mathscr{E})$ the variety $\operatorname{Proj}_{X}\left(\mathrm{~S}^{\bullet} \mathscr{E}\right)$, and by $\mathscr{O}_{\mathbb{P}_{X}(\mathscr{E})}(1)$ its tautological line bundle.

2.5. Reflexive hull. Given a normal variety $X$ and a coherent sheaf $\mathscr{E}$ on $X$, write $\operatorname{det} \mathscr{E}:=\left(\Lambda^{\text {rank } \mathscr{E}} \mathscr{E}\right)^{* *}$. Given any morphism $f: Y \rightarrow X$ of normal varieties, write $f^{[*]} \mathscr{E}:=\left(f^{*} \mathscr{E}\right)^{* *}$.

2.6. Singularities of pairs. A pair $(X, D)$ consists of a normal quasi-projective variety $X$ and a (not necessarily effective) $\mathbb{Q}$-divisor $D$ on $X$. A reduced pair is a pair $(X, D)$ such that $D$ is effective and reduced. We will use the notions of canonical, klt, and log canonical singularities for pairs without further explanation or comment and simply refer to [Kol97] for a discussion and for their precise definitions.

The following elementary fact will be used throughout the paper (see [Kol97, Proposition 3.16]).

Fact 2.3. Let $\gamma: X_{1} \rightarrow X$ be a finite cover between normal complex varieties. Let $D$ be a $\mathbb{Q}$-divisor on $X$, and set $D_{1}:=\gamma^{*}\left(K_{X}+D\right)-K_{X_{1}}$. Then $(X, D)$ is klt (resp. log canonical) if and only $\left(X_{1}, D_{1}\right)$ is klt (resp. log canonical).

We will also need the following definition.

Definition 2.4. A normal, quasi-projective variety $X$ is said to be of $k l t$ type if there exists an effective $\mathbb{Q}$-divisor $D$ on $X$ such that $(X, D)$ is klt.

Remark 2.5. If $X$ is of klt type and $\mathbb{Q}$-factorial, then $X$ has klt singularities.

Lemma 2.6. Let $X$ be a normal projective variety and let $D$ be a reduced effective divisor on $X$ such that $K_{X}+D$ is $\mathbb{Q}$-Cartier. Let $\gamma: X_{1} \rightarrow X$ be a finite cover of normal projective varieties. Suppose that $\gamma$ is quasi-étale over $X \backslash \operatorname{Supp} D$. Then the following holds.

(1) The divisor $D_{1, \varepsilon}:=\gamma^{*}\left(K_{X}+(1-\varepsilon) D\right)-K_{X_{1}}$ is effective if $0 \leqslant \varepsilon \ll 1$. The divisor $D_{1}:=\gamma^{*}\left(K_{X}+\right.$ $D)-K_{X_{1}}$ is (effective and) reduced, and $\gamma^{-1}(\operatorname{Supp} D) \subseteq \operatorname{Supp} D_{1}$.

(2) Suppose in addition that $(X, D)$ is log canonical and that $X$ is $\mathbb{Q}$-factorial with klt singularities. Then $X_{1}$ is of klt type.

Proof. Let $Q$ be a prime divisor on $X_{1}$ and let $m$ be the ramification index of $\gamma$ along $Q$. Set $P:=\gamma(Q)$. A straightforward local computation then shows that

$$
\operatorname{mult}_{Q} D_{1, \varepsilon}=-(m-1)+m(1-\varepsilon) \operatorname{mult}_{P} D .
$$

By assumption, any irreducible component of the branch locus of $\gamma$ which has codimension 1 in $X$ is contained in the support of $D$. Thus, if $P$ is not contained in the support of $D$, then we must have $m=1$. Item (1) follows easily.

Suppose from now on that $(X, D)$ is $\log$ canonical and that $X$ is $\mathbb{Q}$-factorial with klt singularities. Then $(X,(1-\varepsilon) D)$ is klt for any $0<\varepsilon \leqslant 1$. This implies that the pair $\left(X_{1}, D_{1, \varepsilon}\right)$ is klt as well. Item (2) now follows from Item (1), completing the proof of the lemma.

2.7. Logarithmic differential forms. Let $X$ be a smooth variety, and let $D \subset X$ a divisor with simple normal crossings. Let

$$
T_{X}(-\log D) \subseteq T_{X}=\operatorname{Der}_{\mathbb{C}}\left(\mathscr{O}_{X}\right)
$$

be the subsheaf consisting of those derivations that preserve the ideal sheaf $\mathscr{O}_{X}(-D)$. One easily checks that the logarithmic tangent sheaf $T_{X}(-\log D)$ is a locally free sheaf of Lie subalgebras of $T_{X}$, having the same restriction to $X \backslash D$. Set $n:=\operatorname{dim} X$. If $D$ is defined at $x$ by the equation $x_{1} \cdots x_{k}=0$, where $x_{1}, \ldots, x_{k}$ form part of a regular system of parameters $\left(x_{1}, \ldots, x_{n}\right)$ of the local ring $\mathscr{O}_{X, x}$ of $X$ at $x$, then a local basis of $T_{X}(-\log D)$ (after localization at $\left.x\right)$ consists of

$$
x_{1} \partial_{1}, \ldots, x_{k} \partial_{k}, \partial_{k+1}, \ldots, \partial_{n},
$$

where $\left(\partial_{1}, \ldots, \partial_{n}\right)$ is the local basis of $T_{X}$ dual to the local basis $\left(d x_{1}, \ldots, d x_{n}\right)$ of $\Omega_{X}^{1}$.

A local computation shows that $T_{X}(-\log D)$ can be identified with the subsheaf of $T_{X}$ containing those vector fields that are tangent to $D$ at smooth points of $D$. 
The dual of $T_{X}(-\log D)$ is the sheaf $\Omega_{X}^{1}(\log D)$ of logarithmic differential 1-forms. More generally, if $1 \leqslant p \leqslant$ $n$, then $\Omega_{X}^{p}(\log D):=\wedge^{p} \Omega_{X}^{1}(\log D)$ is the sheaf logarithmic differential $p$-forms, that is, of rational $p$-forms $\alpha$ on $X$ such that $\alpha$ and $d \alpha$ have at most simple poles along $D$. The top exterior power $\operatorname{det} \Omega_{X}^{1}(\log D)=\Omega_{X}^{n}(\log D)$ is the invertible sheaf $\mathscr{O}_{X}\left(K_{X}+D\right)$, where $K_{X}$ denotes a canonical divisor.

2.8. Reflexive (logarithmic) differentials forms. Given a normal variety $X$, we denote the sheaf of Kähler differentials by $\Omega_{X}^{1}$. If $0 \leqslant p \leqslant \operatorname{dim} X$ is any integer, write $\Omega_{X}^{[p]}:=\left(\Omega_{X}^{p}\right)^{* *}$. The tangent sheaf $\left(\Omega_{X}^{1}\right)^{*}$ will be denoted by $T_{X}$.

If $D$ is a reduced effective divisor on $X$ we denote by $(X, D)_{\text {reg }}$ the open set where $(X, D)$ is $\log$ smooth. If $1 \leqslant p \leqslant \operatorname{dim} X$ is any integer, we write $\Omega_{X}^{[p]}(\log D)$ for the reflexive sheaf on $X$ whose restriction to $U:=(X, D)_{\text {reg }}$ is the sheaf of logarithmic differential forms $\Omega_{U}^{p}\left(\log D_{\mid U}\right)$. We will refer to it as the sheaf of reflexive logarithmic $p$-forms. Suppose that $X$ is smooth and let $t$ be a defining equation for $D$ on some open set $X^{\circ}$. Let $\alpha$ be a rational $p$-form on $X$. Then $\alpha$ is a reflexive logarithmic $p$-form on $X^{\circ}$ if and only if $t \alpha$ and $t d \alpha$ are regular on $X^{\circ}$ (see [Sai80]).

The dual of $\Omega_{X}^{1}(\log D)$ is logarithmic tangent sheaf $T_{X}(-\log D)$.

Lemma 2.7. Let $\gamma: X_{1} \rightarrow X$ be a finite cover between normal varieties, and let $D$ be a reduced effective divisor on $X$. Let $1 \leqslant p \leqslant \operatorname{dim} X$ be any integer.

(1) If $D_{1}$ is a reduced effective divisor on $X_{1}$ such that $\gamma^{-1}(\operatorname{Supp} D) \subseteq \operatorname{Supp} D_{1}$, then the standard pull-back map of Kähler differentials induces an injective map of reflexive sheaves

$$
\gamma^{[*]} \Omega_{X}^{[p]}(\log D) \hookrightarrow \Omega_{X_{1}}^{[p]}\left(\log D_{1}\right) .
$$

(2) Suppose that $\gamma$ is quasi-étale over $X \backslash \operatorname{Supp} D$ and set $D_{1}:=\gamma^{*}\left(K_{X}+D\right)-K_{X_{1}}$. Then $D_{1}$ is reduced and effective. Moreover, the standard pull-back map of Kähler differentials induces an isomorphism

$$
\gamma^{[*]} \Omega_{X}^{[p]}(\log D) \cong \Omega_{X_{1}}^{[p]}\left(\log D_{1}\right)
$$

Proof. Let $D_{1}$ be a reduced effective divisor on $X_{1}$ such that $\gamma^{-1}(\operatorname{Supp} D) \subseteq \operatorname{Supp} D_{1}$. Let $U \subseteq(X, D)_{\text {reg }}$ be an open set with complement of codimension at least 2 such that $\gamma^{-1}(U) \subseteq\left(X_{1}, D_{1}\right)_{\text {reg. By [KMM87, Lemma }}$ 0.2.13] applied to the restriction of $\gamma$ to $\gamma^{-1}(U)$, the standard pull-back map of Kähler differentials induces an injective map of locally free sheaves

$$
\left(\gamma_{\mid \gamma^{-1}(U)}\right)^{*} \Omega_{U}^{p}\left(\log D_{\mid U}\right) \hookrightarrow \Omega_{\gamma^{-1}(U)}^{p}\left(\log D_{1 \mid \gamma^{-1}(U)}\right) .
$$

This easily implies that there is an injective morphism of reflexive sheaves

$$
\gamma^{[*]} \Omega_{X}^{[p]}(\log D) \hookrightarrow \Omega_{X_{1}}^{[p]}\left(\log D_{1}\right) .
$$

Suppose now that $\gamma$ is quasi-étale over $X \backslash \operatorname{Supp} D$ and set $D_{1}:=\gamma^{*}\left(K_{X}+D\right)-K_{X_{1}}$. By Lemma 2.6, $D_{1}$ is effective and reduced, and $\gamma^{-1}(\operatorname{Supp} D) \subseteq \operatorname{Supp} D_{1}$. A straightforward local computation then shows that the above map yields an isomorphism

finishing the proof of the lemma.

$$
\gamma^{[*]} \Omega_{X}^{[p]}(\log D) \cong \Omega_{X_{1}}^{[p]}\left(\log D_{1}\right)
$$

Lemma 2.8. Let $f: X \rightarrow Y$ be surjective morphism of normal varieties, and let $D$ and $B$ be reduced effective divisors on $X$ and $Y$ respectively. Suppose that Supp $D$ contains all codimension 1 irreducible components of $f^{-1}(\operatorname{Supp} B)$ which are not $f$-exceptional. Then the standard pull-back map of Kähler differentials induces an injective map of reflexive sheaves

for any integer $1 \leqslant p \leqslant \operatorname{dim} X$.

$$
\Omega_{Y}^{[p]}(\log B) \rightarrow\left(f_{*} \Omega_{X}^{[p]}(\log D)\right)^{* *}
$$

Proof. Let $C$ be the reduced effective divisor on $X$ whose support is the union of the codimension 1 irreducible components of $f^{-1}(\operatorname{Supp} B)$ which are not $f$-exceptional. Let $V \subseteq(Y, B)_{\text {reg }}$ be the complement of the images of $f$-exceptional divisors contained in $f^{-1}(\operatorname{Supp} B)$, and set $U:=(X, C)_{\text {reg }} \cap f^{-1}(V)$. By assumption, Supp $C \subseteq$ Supp D. By [Del70, Chapitre 2, Proposition 3.2] applied to the restriction of $f$ to $U$, the standard pull-back map of Kähler differentials induces an injective map of sheaves

$$
\Omega_{V}^{p}\left(\log B_{\mid V}\right) \rightarrow\left(f_{\mid U}\right)_{*} \Omega_{U}^{p}\left(\log C_{\mid U}\right)
$$


for any $1 \leqslant p \leqslant \operatorname{dim} Y$. On the other hand, since $D-C$ is effective by assumption, we have a natural map

$$
\left(f_{\mid U}\right)_{*} \Omega_{U}^{p}\left(\log C_{\mid U}\right) \rightarrow\left(f_{\mid U}\right)_{*} \Omega_{U}^{p}\left(\log D_{\mid U}\right)=\left(f_{\mid f^{-1}(V)}\right)_{*} \Omega_{f^{-1}(V)}^{[p]}\left(\log D_{\mid f^{-1}(V)}\right) .
$$

This easily implies that there is a morphism

$$
\Omega_{Y}^{[p]}(\log B) \rightarrow\left(f_{*} \Omega_{X}^{[p]}(\log D)\right)^{* *},
$$

finishing the proof of the lemma.

2.9. Resolution of singularities. We will consider a suitable resolution of singularities of a given variety whose existence is guaranteed by the following theorem.

Theorem 2.9 ([GKK10, Corollary 4.7]). Let $X$ be a normal variety and let $D$ be a reduced effective divisor on $X$. Then there exists a $\log$ resolution $\beta: Y \rightarrow X$ of $(X, D)$ such that $\beta_{*} T_{Y}(-\log E) \cong T_{X}(-\log D)$, where $E$ is the largest reduced divisor contained in $\beta^{-1}(\operatorname{Supp} D)$.

We call a resolution $\beta$ as in Theorem 2.9 a canonical resolution of the pair $(X, D)$. In the course of the proof of our main result, we will need the following observation (see [Ber17, Corollary 3.5] for a somewhat related result).

Lemma 2.10. Let $(X, D)$ be a log canonical pair with $D$ effective and reduced. Let $\beta: Y \rightarrow X$ be a canonical resolution of $(X, D)$ and let $E$ be the largest reduced divisor contained in $\beta^{-1}(\operatorname{Supp} D)$. Suppose that $\Omega_{X}^{[1]}(\log D)$ is locally free. Then $\beta^{*} \Omega_{X}^{[1]}(\log D) \cong \Omega_{Y}^{1}(\log E)$. In particular, $\Omega_{Y}^{1}(\log E)$ is locally free.

Proof. The morphism of locally free sheaves

$$
T: \beta^{*} T_{X}(\log D) \cong \beta^{*}\left(\beta_{*} T_{Y}(\log E)\right) \rightarrow T_{Y}(\log E)
$$

yields a map

$$
\operatorname{det} T: \beta^{*} \mathscr{O}_{X}\left(-K_{X}-D\right) \cong \beta^{*} \operatorname{det} T_{X}(\log D) \rightarrow \operatorname{det} T_{Y}(\log E) \cong \mathscr{O}_{Y}\left(-K_{Y}-E\right),
$$

which is an isomorphism over $Y \backslash \beta(\operatorname{Exc} \beta)$. Since $(X, D)$ is $\log$ canonical, $\operatorname{det} T$ must be an isomorphism. This immediately implies that $T$ is an isomorphism as well, proving the lemma.

2.10. R-flat vector bundles. For the reader's convenience, we recall the notion of numerical flatness for vector bundles.

Definition 2.11. Let $f: X \rightarrow Y$ be a projective morphism of quasi-projective varieties. A locally free sheaf $\mathscr{E}$ on $X$ of positive rank is called $f$-numerically flat if $\mathscr{E}$ and $\mathscr{E}^{*}$ are $f$-nef. If $Y$ is a point, we simply say that $\mathscr{E}$ is numerically flat.

In the course of the proof of [BdS09, Theorem 1.1] the authors show that a locally free sheaf $\mathscr{E}$ of positive rank $r$ on a smooth projective rationally connected variety $X$ such that $\nu^{*} \mathscr{E} \cong \mathscr{O}_{\mathbb{P}^{1}}^{\oplus r}$ for any morphism $\nu: \mathbb{P}^{1} \rightarrow X$ is numerically flat. Definition 2.12 is a formalization of the above condition.

Definition 2.12. Let $f: X \rightarrow Y$ be a projective morphism of quasi-projective varieties. A locally free sheaf $\mathscr{E}$ on $X$ of positive rank $r$ is called relatively R-flat or $f$-relatively R-flat if $\nu^{*} \mathscr{E} \cong \mathscr{O}_{\mathbb{P 1}}^{\oplus r}$ for any morphism $\nu: \mathbb{P}^{1} \rightarrow X$ such that $\nu\left(\mathbb{P}^{1}\right)$ is contracted by $f$. If $Y$ is a point, we simply say that $\mathscr{E}$ is R-flat.

The following result partly extends [BdS09, Theorem 1.1] to our setting.

Lemma 2.13. Let $X$ be a projective reduced space, not necessarily irreducible. Suppose that $X$ is rationally chain connected. Then any locally free, $R$-flat sheaf on $X$ is numerically flat.

Proof. Let $\mathscr{E}$ be a locally free, R-flat sheaf on $X$. Let $C$ be a curve on $X$ and let $x \in X$ be a general point. Since $X$ is rationally chain connected, there exist finitely many normal projective surfaces $S_{i}(1 \leqslant i \leqslant N)$ as well as surjective morphisms $\pi_{i}: S_{i} \rightarrow B_{i}$ onto smooth complete curves such that the following holds. The general fibers of $\pi_{i}$ are rational curves. Moreover, there exist sections $\sigma_{i, 1} \subset S_{i}$ and $\sigma_{i, 2} \subset S_{i}$ of $\pi_{i}$ and morphisms $e_{i}: S_{i} \rightarrow X$ such that $e_{1}\left(\sigma_{1,1}\right)=\{x\}, e_{i}\left(\sigma_{i, 2}\right)=e_{i+1}\left(\sigma_{i+1,1}\right)$ for $1 \leqslant i \leqslant N-1$ and $e_{N}\left(\sigma_{N, 2}\right)=C$. By the semistable reduction theorem, we may assume without loss of generality that $\pi_{i}$ is semistable. In particular, any fiber of $\pi_{i}$ is a rational tree. Notice also that $\pi_{i}$ is flat. Since $\mathscr{E}$ is R-flat, we must have $e_{i}^{*} \mathscr{E} \cong \pi_{i}^{*} \mathscr{G}_{i}$ for some locally free sheaf $\mathscr{G}_{i}$ on $B_{i}$. Now, $e_{1}^{*} \mathscr{E}_{\mid \sigma_{1,1}}$ is trivial since $e_{1}\left(\sigma_{1,1}\right)=\{x\}$. It follows that $\mathscr{G}_{1}$ is numerically flat and hence so is $e_{1}^{*} \mathscr{E}$. This in turn implies that $\mathscr{E}_{\mid e_{1}\left(\sigma_{1,2}\right)}=\mathscr{E}_{\mid e_{2}\left(\sigma_{2,1}\right)}$ is numerically flat as well if $\operatorname{dim} e_{1}\left(\sigma_{1,2}\right)=1$. If $\operatorname{dim} e_{1}\left(\sigma_{1,2}\right)=0$, then $\mathscr{E}_{\mid e_{1}\left(\sigma_{1,2}\right)}=\mathscr{E}_{\mid e_{2}\left(\sigma_{2,1}\right)}$ is the trivial vector bundle. An induction on $i$ then shows that $\mathscr{E}_{\mid C}$ is numerically flat, finishing the proof of the lemma. 
The following lemma will be very useful.

Lemma 2.14. Let $f: X \rightarrow Y$ be a projective morphism of quasi-projective varieties, and let $\mathscr{E}$ be a locally free sheaf on $Y$. Suppose that $X$ is of klt type and that $-K_{X}$ is $f$-ample. If $f^{*} \mathscr{E}$ is $R$-flat, then so is $\mathscr{E}$.

Proof. This is an immediate consequence of [HM07, Corollary 1.10].

2.11. Discriminant. Let $f: X \rightarrow Y$ be a projective morphism with connected fibers of normal, quasi-projective varieties. Let $D$ be a $\mathbb{Q}$-divisor on $X$ such that $(X, D)$ is $\log$ canonical over the generic point of $Y$. The discriminant divisor of $(f, D)$ is the $\mathbb{Q}$-divisor $B=\sum_{P} b_{P} P$ on $Y$, where $P$ runs through all prime divisor on $Y$, and

$$
1-b_{P}:=\sup \left\{t \in \mathbb{R} \mid\left(X, D+t f^{*} P\right) \text { is } \log \text { canonical over the generic point of } P\right\} \text {. }
$$

The discriminant divisor measures the singularities of special fibers. For the reader's convenience, we recall three standard facts.

Fact 2.15. If $C$ is a $\mathbb{Q}$-divisor on $Y$, then the discriminant divisor of $\left(f, D+f^{*} C\right)$ is $B+C$.

Fact 2.16 ([Amb04, Lemma 2.6]). Suppose that $K_{X}+D$ is $\mathbb{Q}$-Cartier. Let $\beta: Z \rightarrow X$ be a projective birational morphism with $Z$ normal. Set $g:=f \circ \beta$ and $C:=\beta^{*}\left(K_{X}+D\right)-K_{Z}$. Then the discriminant divisor of $(g, C)$ is $B$.

Fact 2.17 ([Amb04, Lemma 5.1]). Let $\gamma: Y_{1} \rightarrow Y$ be a finite morphism with $Y_{1}$ normal, and let $X_{1}$ be the normalization of the product $Y_{1} \times_{X} X_{1}$ with natural morphisms $f_{1}: X_{1} \rightarrow Y_{1}$ and $\gamma_{1}: X_{1} \rightarrow X$. Set $D_{1}:=$ $\gamma_{1}^{*}\left(K_{X}+D\right)-K_{X_{1}}$. Let $B_{1}$ be the discriminant divisor of $\left(f_{1}, D_{1}\right)$. Then $K_{X_{1}}+B_{1} \sim_{\mathbb{Z}} \gamma_{1}^{*}\left(K_{X}+B\right)$.

We will also need the following observation.

Lemma 2.18. Let $X$ be a normal projective variety, and let $D$ be an effective $\mathbb{Q}$-divisor on $X$. Suppose that $(X, D)$ has klt singularities. Let $\psi: X \rightarrow Y$ be a projective morphism with connected fibers onto a normal, projective variety $Y$, and let $B$ the discriminant divisor of $(\psi, D)$. Suppose that $-\left(K_{X}+D\right)$ is $\psi$-ample. If $K_{Y}+B$ is $\mathbb{Q}$-Cartier, then $(Y, B)$ has klt singularities.

Proof. Let $H_{Y}$ be an ample $\mathbb{Q}$-divisor on $Y$ such that $H_{X}:=-\left(K_{X}+D\right)+\psi^{*} H_{Y}$ is ample, and let $m \geqslant 2$ be an integer such that $m H_{X}$ is very ample. Let $D_{1} \in\left|m H_{X}\right|$ be a general member. By general choice of $D_{1}$, we may assume that $\left(X, D+\frac{1}{m} D_{1}\right)$ has klt singularities and that the discriminant divisor of $\left(\psi, D+\frac{1}{m} D_{1}\right)$ is $B$. Notice that $K_{X}+D+\frac{1}{m} D_{1} \sim_{\mathbb{Q}} \psi^{*} H_{Y}$. Arguing as in the proof of [Fuj99, Theorem 1.2], one then shows that there exists an effective divisor $B_{1}$ on $Y$ such that $\left(Y, B_{1}\right)$ is klt and $B \leqslant B_{1}$. This immediately implies that $(Y, B)$ is klt, proving the lemma.

\section{A CANONICAL BUNDLE FORMULA FOR GENERICALLY ISOTRIVIAL FIBRATIONS}

In this section we prove a canonical bundle formula for generically isotrivial fibrations (see Theorem 3.3), which might be of independent interest. The canonical bundle formula for the so-called lc-trivial fibrations is a higher dimensional analogue of Kodaira's canonical bundle formula for minimal elliptic surfaces. We refer to [Kol07] and the references therein for a (rather delicate) precise formulation.

We will use the following definition.

Definition 3.1. Let $f: X \rightarrow Y$ be a morphism with connected fibers of algebraic varieties, and let $D$ be an integral divisor on $X$. Suppose that $D$ is effective and reduced over the generic point of $Y$. The fibration $(f, D)$ is called generically isotrivial if there is a dense Zariski open set $Y^{\circ} \subseteq Y$ such that the following holds. For every point $y \in Y^{\circ}$, there exists a Euclidean open neighbourhood $U$ of $y$ in $Y^{\circ}$ such that

$$
\left(f^{-1}(U), D_{\mid f^{-1}(U)}\right) \cong\left(U \times F, U \times D_{\mid F}\right)
$$

over $U$, where $F$ if a fiber of $f_{\mid f^{-1}(U)}$.

Remark 3.2. Setup as in Definition 3.1. Suppose in addition that $f$ is projective. Then $(f, D)$ is generically isotrivial if and only if there exists a dense Zariski open set $Y^{\circ} \subseteq Y$ and a finite morphism $\gamma^{\circ}: Y_{1}^{\circ} \rightarrow Y^{\circ}$ such that

$$
\left(X_{1}^{\circ}, D_{1 \mid X_{1}^{\circ}}^{\circ}\right) \cong\left(Y_{1}^{\circ} \times F_{1}, Y_{1}^{\circ} \times D_{1 \mid F_{1}}^{\circ}\right)
$$

over $Y_{1}^{\circ}$, where $X_{1}^{\circ}$ denotes the normalization of $Y_{1}^{\circ} \times_{Y} \circ X$ with natural morphisms $f_{1}^{\circ}: X_{1}^{\circ} \rightarrow Y_{1}^{\circ}$ and $\gamma_{1}^{\circ}: X_{1}^{\circ} \rightarrow$ $X^{\circ}=: f^{-1}\left(Y^{\circ}\right), F_{1}$ is a general fiber of $f_{1}^{\circ}$, and $D_{1}^{\circ}:=\left(\gamma_{1}^{\circ}\right)^{*}\left(D_{\mid X^{\circ}}\right)$. Indeed, shrinking $Y$, if necessary, we may 
assume that $f$ is flat and that the restriction of $f$ to any irreducible component of $D$ is also flat over $Y$. The claim is then an easy consequence of the fact that the $\operatorname{space}^{\operatorname{Isom}_{Y}}\left((X, D),\left(Y \times F, Y \times D_{\mid F}\right)\right)$ parametrizing isomorphims of pairs $\left(X_{y}, D_{\mid X_{y}}\right) \cong\left(F, D_{\mid F}\right)$ with $y \in Y$ is quasi-projective over $Y$.

Theorem 3.3. Let $X$ be a normal projective variety, and let $f: X \rightarrow Y$ be a surjective morphism with connected fibers onto a normal projective variety $Y$. Let also $D$ be a Weil divisor on $X$. Suppose that $D$ is effective in a neighbourhood of a general fiber of $f$ and that $(X, D)$ is log canonical over the generic point of $Y$. Suppose in addition that there exists a Cartier divisor $C$ on $Y$ such that $K_{X}+D \sim_{\mathbb{Q}} f^{*} C$. If $(f, D)$ is generically isotrivial, then $C \sim_{\mathbb{Q}} K_{Y}+B$, where $B$ denotes the discriminant divisor of $(f, D)$.

Proof. Let $m$ be smallest positive integer such that $m\left(K_{X}+D\right) \sim_{\mathbb{Z}} m f^{*} C$, and let $\gamma: X_{1} \rightarrow X$ be the corresponding index one cover, which is quasi-étale (see [KM98, Definition 2.52]). Set $D_{1}:=\gamma^{*}\left(K_{X}+D\right)-K_{X_{1}}$. By choice of $m$, the morphism $f_{1}:=f \circ \gamma$ has connected fibers. Notice that $D_{1}$ is effective in a neighbourhood of a general fiber of $f_{1}$ and that the pair $\left(X_{1}, D_{1}\right)$ is $\log$ canonical over the generic point of $Y$. Moreover, by construction, $K_{X_{1}}+D_{1} \sim_{\mathbb{Z}} f_{1}^{*} C$. By Fact $2.16, B$ is also the discriminant divisor of $\left(f_{1}, D_{1}\right)$. Finally, the fibration $\left(f_{1}, D_{1}\right)$ is easily seen to be generically isotrivial as well using Lemma 3.5 below. Replacing $(f, D)$ by $\left(f_{1}, D_{1}\right)$, if necessary, we may therefore assume without loss of generality that the following holds.

Assumption 3.4. The relation $K_{X}+D \sim_{\mathbb{Z}} f^{*} C$ holds. In particular, $K_{X}+D$ is Cartier.

By assumption (see also Remark 3.2), there exists a finite cover $\gamma: Y_{1} \rightarrow Y$ such that the following holds. Let $X_{1}$ be the normalization of $Y_{1} \times_{Y} X$ with natural morphisms $f_{1}: X_{1} \rightarrow Y_{1}$ and $\tau: X_{1} \rightarrow X$. Set $D_{1}:=$ $\tau^{*}\left(K_{X}+D\right)-K_{X_{1}}$ and let $F_{1}$ be a general fiber of $f_{1}$. Then there exist a dense open set $Y_{1}^{\circ} \subseteq Y_{1}$ and an isomorphism of pairs

$$
\left(f_{1}^{-1}\left(Y_{1}^{\circ}\right), D_{1 \mid f^{-1}\left(Y_{1}^{\circ}\right)}\right) \cong\left(Y_{1}^{\circ} \times F_{1}, Y_{1}^{\circ} \times D_{1 \mid F_{1}}\right)
$$

over $Y_{1}^{\circ}$. Let $B_{1}$ denotes the discriminant divisor of $\left(f_{1}, D_{1}\right)$. Let $\gamma_{1}: Y_{2} \rightarrow Y_{1}$ be a resolution of singularities, and let $\tau_{1}: X_{2} \rightarrow X_{1}$ be a resolution of the main component of the product $Y_{2} \times_{Y_{1}} X_{1}$ with natural morphism $f_{2}: X_{2} \rightarrow Y_{2}$. Set $D_{2}:=\tau_{1}^{*}\left(K_{X_{1}}+D_{1}\right)-K_{X_{2}}$. Set also $Y_{2}^{\circ}:=\gamma_{1}^{-1}\left(Y_{1}^{\circ}\right)$ and $X_{2}^{\circ}:=f_{2}^{-1}\left(Y_{2}^{\circ}\right)$. We may assume without loss of generality that $Y_{2} \backslash Y_{2}^{\circ}$ has simple normal crossings and that

$$
\left(X_{2}^{\circ}, D_{2 \mid X_{2}^{\circ}}\right) \cong\left(Y_{2}^{\circ} \times F_{2}, Y_{2}^{\circ} \times D_{2 \mid F_{2}}\right)
$$

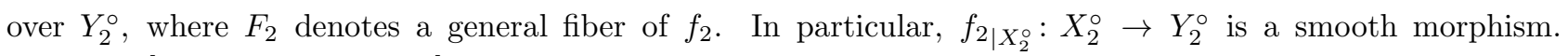
Applying [Vie95, Theorem 6.4], we see that there exists a finite morphism $\gamma_{2}: Y_{3} \rightarrow Y_{2}$ of complex manifolds and a resolution $X_{4}$ of the normalization $X_{3}$ of the product $Y_{3} \times_{Y_{2}} X_{2}$ such that the following holds. Let $f_{4}: X_{4} \rightarrow Y_{3}$ denote the natural morphism, and set $Y_{3}^{\circ}:=\gamma_{2}^{-1}\left(Y_{2}^{\circ}\right)$. Then $Y_{3} \backslash Y_{3}^{\circ}$ and $f_{4}^{-1}\left(Y_{3} \backslash Y_{3}^{\circ}\right)$ have simple normal crossings, and $f_{4}$ has reduced fibers in codimension one. We obtain a commutative diagram as follows:

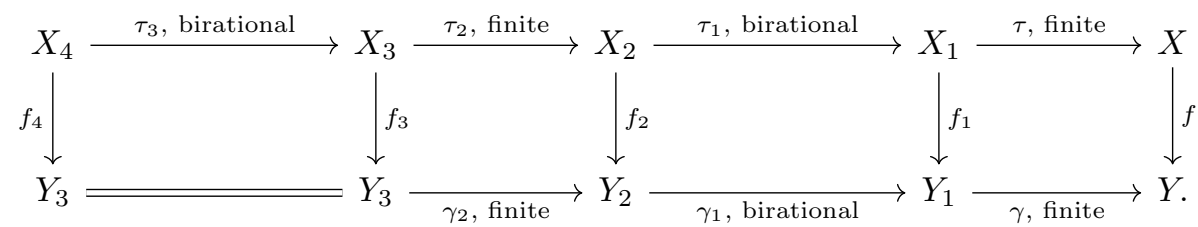

Set $D_{3}:=\tau_{2}^{*}\left(K_{X_{2}}+D_{2}\right)-K_{X_{3}}$ and $D_{4}:=\tau_{3}^{*}\left(K_{X_{3}}+D_{3}\right)-K_{X_{4}}$. Notice that $D_{4}$ has integral coefficients since $K_{X}+D$ is Cartier by assumption. Set also $X_{4}^{\circ}:=f_{4}^{-1}\left(Y_{3}^{\circ}\right)$. Finally, we may assume without loss of generality that $X_{4}$ is a $\log$ resolution of $\left(X_{3}, D_{3}\right)$ and that

$$
\left(X_{4}^{\circ}, D_{4 \mid X_{4}^{\circ}}\right) \cong\left(Y_{3}^{\circ} \times F_{4}, Y_{3}^{\circ} \times D_{4 \mid F_{4}}\right)
$$

over $Y_{3}^{\circ}$, where $F_{4}$ denotes a general fiber of $f_{4}$.

Write $D_{4}=R_{4}+S_{4}-E_{4}-G_{4}$, where $R_{4}, S_{4}, E_{4}$ and $G_{4}$ are effective divisors with no common components such that any irreducible component of $R_{4}+E_{4}$ maps onto $Y_{3}$ and any irreducible component of $S_{4}+G_{4}$ maps into a proper subset of $Y_{3}$. Observe that the divisor $R_{4}$ is reduced since $(X, D)$ is log canonical over the generic point of $Y$. Moreover, since $D$ is effective in a neighbourhood of a general fiber of $f$, any irreducible component of $E_{4}$ is exceptional over $X_{1}$. Blowing-up strata of $R_{4}$ mapping into a proper subset of $Y_{3}$, if necessary, we may also assume that any stratum of $R_{4}$ dominates $Y_{3}$. Set $f_{4}^{\circ}:=f_{4 \mid X_{4}^{\circ} \backslash \operatorname{Supp}\left(R_{4}\right)}$ and $d:=\operatorname{dim} X_{4}-\operatorname{dim} Y_{3}$. By construction, the local system $\left(R^{d} f_{4}^{\circ}\right)_{*} \mathbb{C}_{X_{4}^{\circ} \backslash \operatorname{Supp}\left(R_{4}\right)}$ is trivial. In particular, the Deligne's canonical extension of $\left(R^{d} f_{4}^{\circ}\right)_{*} \mathbb{C}_{X_{4}^{\circ} \backslash \operatorname{Supp}\left(R_{4}\right)}$ is the trivial local system on $Y_{3}$ (see [Del70, pp. 91-95]). Now, by [Fuj04, Theorems 
3.1 and 3.9], the sheaf $\left(f_{4}\right)_{*} \mathscr{O}\left(K_{X_{4} / Y_{3}}+R_{4}\right)$ is locally free (of rank 1) and numerically effective. Moreover, it identifies with the so-called (upper) canonical extension of

$$
F^{d}\left(\left(R^{d} f_{4}^{\circ}\right)_{*} \mathbb{C}_{X_{4}^{\circ} \backslash \operatorname{Supp}\left(R_{4}\right)} \otimes \mathscr{O}_{Y_{3}^{\circ}}\right) \subset\left(R^{d} f_{4}^{\circ}\right)_{*} \mathbb{C}_{X_{4}^{\circ} \backslash \operatorname{Supp}\left(R_{4}\right)} \otimes \mathscr{O}_{Y_{3}^{\circ}},
$$

where $F^{\bullet}\left(\left(R^{d} f_{4}^{\circ}\right)_{*} \mathbb{C}_{X_{4}^{\circ} \backslash \operatorname{Supp}\left(R_{4}\right)} \otimes \mathscr{O}_{Y_{3}^{\circ}}\right)$ denotes the Hodge filtration (see [Fuj04, Section 3.1] and the references therein). This immediately implies that

$$
\left(f_{4}\right)_{*} \mathscr{O}\left(K_{X_{4} / Y_{3}}+R_{4}\right) \cong \mathscr{O}_{Y_{3}},
$$

and hence

$$
\mathscr{O}_{Y_{3}}\left(C_{3}\right) \otimes\left(f_{4}\right)_{*} \mathscr{O}_{X_{4}}\left(E_{4}+G_{4}-S_{4}\right) \cong \mathscr{O}_{Y_{3}}\left(K_{Y_{3}}\right)
$$

where $C_{3}$ denotes the pull-back of $C$ to $Y_{3}$.

Let $B_{3}^{+}$be the smallest effective divisor on $Y_{3}$ such that $S_{4} \leqslant f_{4}^{*} B_{3}^{+}$over the codimension 1 points of $f_{4}\left(\operatorname{Supp} S_{4}\right)$ and let $B_{3}^{-}$be the largest (effective) divisor on $Y_{3}$ such that $\operatorname{Supp} B_{3}^{-} \subseteq f_{4}\left(\operatorname{Supp} G_{4}\right)$ and $f_{4}^{*} B_{3}^{-} \leqslant$ $G_{4}$ over the codimension 1 points of $f_{4}\left(\operatorname{Supp} G_{4}\right)$. By construction, there is an open set $W \subseteq Y_{3}$ with complement of codimension at least 2 and a natural inclusion

$$
\mathscr{O}_{Y_{3}}\left(B_{3}^{-}-B_{3}^{+}\right)_{\mid W} \subseteq\left(f_{4}\right)_{*} \mathscr{O}_{X_{4}}\left(E_{4}+G_{4}-S_{4}\right)_{\mid W} .
$$

We now show that this map is an isomorphism. Let $U \subseteq W$ be dense open set, and let $t$ be a rational function on $X_{4}$ such that

$$
(\operatorname{div} t)_{\mid f_{4}^{-1}(U)}+\left(E_{4}+G_{4}-S_{4}\right)_{\mid f_{4}^{-1}(U)} \geqslant 0 .
$$

Since $E_{4}$ is exceptional over $X_{1}$, any regular function on $F_{4} \backslash \operatorname{Supp} E_{4 \mid F_{4}}$ is constant. This immediately implies that

Moreover, since

$$
(\operatorname{div} t)_{\mid f_{4}^{-1}(U)}+\left(G_{4}-S_{4}\right)_{\mid f_{4}^{-1}(U)} \geqslant 0 .
$$

$$
\left(X_{4}^{\circ}, D_{4 \mid X_{4}^{\circ}}\right) \cong\left(Y_{3}^{\circ} \times F_{4}, Y_{3}^{\circ} \times D_{4 \mid F_{4}}\right)
$$

there exists a rational function $r$ on $Y$ such that $t=r \circ f_{4}$. One then easily checks that

$$
(\operatorname{div} r)_{U}+\left(B_{3}^{-}-B_{3}^{+}\right)_{\mid U} \geqslant 0
$$

using the fact that $f_{4}$ has reduced fibers in codimension 1 . This shows that

$$
\mathscr{O}_{Y_{4}}\left(B_{3}^{-}-B_{3}^{+}\right)_{\mid W} \cong\left(f_{4}\right)_{*} \mathscr{O}_{X_{4}}\left(E_{4}+G_{4}-S_{4}\right)_{\mid W},
$$

and hence

$$
\left(f_{4}\right)_{*} \mathscr{O}_{X_{4}}\left(E_{4}+G_{4}-S_{4}\right) \cong \mathscr{O}_{Y_{3}}\left(B_{3}^{-}-B_{3}^{+}\right)
$$

since both sheaves are locally free on $Y_{3}$.

Next, we show that the discriminant divisor $B_{4}$ of $\left(f_{4}, D_{4}\right)$ is $B_{3}^{+}-B_{3}^{-}$. Let $P$ be a prime divisor on $Y$. If $P$ is not contained in $f_{4}\left(\operatorname{Supp} G_{4}\right) \cup f_{4}\left(\operatorname{Supp} S_{4}\right)$, then $P$ is obviously not contained in the supports of $B_{3}^{+}$and $B_{3}^{-}$. Moreover, $P$ is not contained in the support of $B_{4}$ since $R_{4}$ is a relative normal crossings divisor over $Y_{3} \backslash Y_{3}^{\circ}$ by construction. Suppose that $P$ is contained in $f_{4}\left(\operatorname{Supp} G_{4}\right) \cup f_{4}\left(\operatorname{Supp} S_{4}\right)$. Then $\operatorname{mult}_{P}\left(B_{3}^{-}\right)=0$ since $S_{4}$ and $G_{4}$ have no common components. One then readily checks that $\operatorname{mult}_{P}\left(B_{4}\right)=\operatorname{mult}_{P}\left(B_{3}^{+}-B_{3}^{-}\right)$using the fact that $f_{4}$ has reduced fibers in codimension 1 . This shows that the discriminant divisor of $\left(f_{4}, D_{4}\right)$ is $B_{3}^{+}-B_{3}^{-}$ and thus,

$$
C_{3} \sim_{\mathbb{Z}} K_{Y_{3}}+B_{4} .
$$

By Fact 2.16, we have $B_{4}=B_{3}$. On the other hand, by Fact $2.17, K_{Y_{1}}+B_{1} \sim_{\mathbb{Q}} \gamma^{*}\left(K_{Y}+B\right)$ and $K_{Y_{3}}+B_{3} \sim_{\mathbb{Q}}$ $\gamma_{2}^{*}\left(K_{Y_{2}}+B_{2}\right)$. Finally, $\left(\gamma_{1}\right)_{*} B_{2}$ is obviously the discriminant divisor of $\left(f_{1} \circ \tau_{1}, D_{2}\right)$, and hence $\left(\gamma_{1}\right)_{*} B_{2}=B_{Y_{1}}$ by Fact 2.16 again. This shows that

completing the proof of the theorem.

$$
C \sim_{\mathbb{Q}} K_{Y}+B
$$

Lemma 3.5. Let $X$ be a normal variety and let $d$ be a positive integer. Then there are only finitely many Galois quasi-étale covers of $X$ of degree d up to isomorphism.

Proof. By the Nagata-Zariski purity theorem, any quasi-étale cover of $X$ branches only on the singular set of $X$. Thus, by the Riemann existence theorem (see [sga03, Exposé XII, Théorème 5.1]), we need to show that there are only finitely many normal subgroups of the topological fundamental group $\pi_{1}\left(X_{\text {reg }}\right)$ of index $d$. But this follows easily from the fact that the group $\pi_{1}\left(X_{\text {reg }}\right)$ is finitely generated, proving the lemma. 
The following is an immediate consequence of Theorem 3.3.

Corollary 3.6. Let $X$ be a normal projective variety, and let $f: X \rightarrow Y$ be a surjective morphism with connected fibers onto a normal projective variety $Y$. Let $D$ be a reduced effective divisor on $X$ such that $(X, D)$ is log canonical with $K_{X}+D \sim_{\mathbb{Q}} 0$. Suppose furthermore that $(f, D)$ is generically isotrivial. Then $K_{Y}+B \sim \mathbb{Q} 0$, where $B$ denotes the discriminant divisor of $(f, D)$.

\section{Descent of Vector BUNDLES}

The proof of Theorem 1.9 relies on the following auxiliary statement. To put the result into perspective, consider a Mori extremal contraction $f: X \rightarrow Y$ of a projective klt space. By the cone theorem, a line bundle on $X$ of degree zero on every contracted rational curve comes from $Y$. We first generalize this result to vector bundles of arbitrary rank. The special case where $\operatorname{dim} X=\operatorname{dim} Y$ follows from [GKPT19, Theorem 4.1] together with Lemma 2.13.

Theorem 4.1. Let $f: X \rightarrow Y$ be a projective morphism with connected fibers of normal, quasi-projective varieties. Suppose that there is an effective $\mathbb{Q}$-divisor $D$ on $X$ such that the pair $(X, D)$ is $k l t$ and $-\left(K_{X}+D\right)$ is $f$-ample. Let $\mathscr{E}$ be a locally free, $f$-relatively $R$-flat sheaf on $X$. Then there exists a locally free sheaf $\mathscr{G}$ on $Y$ such that $\mathscr{E} \cong f^{*} \mathscr{G}$.

Proof. By [HM07, Theorem 1.2], every fiber of $f$ is rationally chain connected. Together with Lemma 2.13, this implies that $\mathscr{E}$ is $f$-numerically flat.

If $\operatorname{dim} X=\operatorname{dim} Y$, then Theorem 4.1 follows from [GKPT19, Theorem 4.1]. Suppose from now on that $\operatorname{dim} Y<\operatorname{dim} X$. The proof is similar to that of loc. cit. and so we leave some easy details for the reader.

Let $\mathscr{O}_{\mathbb{P}_{X}(\mathscr{E})}(1)$ denote the tautological line bundle on $\mathbb{P}_{X}(\mathscr{E})$, and let $p: \mathbb{P}_{X}(\mathscr{E}) \rightarrow X$ denote the projection map. Let $C$ be a divisor on $X$ such that $\mathscr{O}_{\mathbb{P}_{X}(\mathscr{E})}(C) \cong \mathscr{O}_{\mathbb{P}_{X}(\mathscr{E})}(1)$ and set $r:=$ rank $\mathscr{E}$. Note that the pair $\left(\mathbb{P}_{X}(\mathscr{E}), p^{*} D\right)$ is klt.

Set $\psi:=f \circ p$, and let $F$ be a general fiber of $f$. Note that $F$ is of klt type and rationally chain connected. By [BdS09, Theorem 1.1] applied to a resolution of $F$ together with [HM07, Theorem 1.2], we must have $\mathscr{E}_{\mid F} \cong \mathscr{O}_{F}^{\oplus r}$. Moreover, by the adjunction formula, $-\left(K_{F}+D_{\mid F}\right)$ is ample. This implies that the restriction of the $\mathbb{Q}$-divisor

$$
C-\left(K_{\mathbb{P}_{X}(\mathscr{E})}+p^{*} D\right) \sim_{\mathbb{Z}}(r+1) \cdot C-p^{*}\left(K_{X}+D+c_{1}(\mathscr{E})\right)
$$

to $G:=p^{-1}(F) \cong \mathbb{P}^{r-1} \times F$ is ample. On the other hand, $C$ is $\psi$-nef since $\mathscr{E}$ is $f$-numerically flat. By the basepoint-free theorem (see [KMM87, Theorem 3.1.1 and Remark 3.1.2]), we conclude that there is a factorization of $\psi$ via a normal variety $Z$

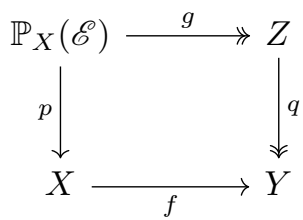

such that $g$ has connected fibers and such that $\mathscr{O}_{\mathbb{P}_{X}(\mathscr{E})}(1)$ is the pull-back of a $q$-ample line bundle $\mathscr{L}$ on $Z$. By construction, the restriction of $\mathscr{O}_{\mathbb{P}_{X}(\mathscr{E})}(1)$ to $G=\psi^{-1}(F) \cong \mathbb{P}^{r-1} \times F$ is isomorphic to the pull-back of $\mathscr{O}_{\mathbb{P}^{r-1}}(1)$ to $\mathbb{P}^{r-1} \times F$ via the projection $\mathbb{P}^{r-1} \times F \rightarrow \mathbb{P}^{r-1}$. This easily implies that

$$
\operatorname{dim} Z=\operatorname{dim} Y+r-1 \text {. }
$$

Moreover, a general fiber of $q$ is isomorphic to $\mathbb{P}^{r-1}$ and the restriction of $\mathscr{L}$ to this fiber is isomorphic to $\mathscr{O}_{\mathbb{P}^{r-1}}(1)$.

Next, we show that $q$ is equidimensional of relative dimension $r-1$. We argue by contradiction and assume that there exists a variety $T \subseteq Z$ with $\operatorname{dim} T=r$ and $\operatorname{dim} q(T)=0$. Let $F_{1}$ be an irreducible component of $f^{-1}(q(T))$ such that the restriction of $g$ to $p^{-1}\left(F_{1}\right)$ induces a surjective morphism $p^{-1}\left(F_{1}\right) \rightarrow T$. We obtain a diagram as follows:

$$
\begin{gathered}
p^{-1}\left(F_{1}\right)=\mathbb{P}_{F_{1}}\left(\mathscr{E}_{\mid F_{1}}\right) \stackrel{g_{p-1}\left(F_{1}\right)}{\longrightarrow} T T \\
p_{\mid p^{-1}\left(F_{1}\right)} \downarrow \\
F_{1} .
\end{gathered}
$$


Since $\mathscr{L}$ is $q$-ample, we have $\left(\mathscr{L}_{\mid T}\right)^{r} \neq 0$. Now, by construction, the pull-back of $\mathscr{L}_{\mid T}$ to $p^{-1}\left(F_{1}\right)$ identifies with the tautological line bundle $\mathscr{O}_{\mathbb{P}_{F_{1}}\left(\mathscr{E}_{\mid F_{1}}\right)}(1)$. On the other hand, $\mathscr{E}_{\mid F_{1}}$ is numerically flat. Thus, by [DPS94, Corollary 1.19] applied to the pull-back of $\mathscr{E}_{\mid F_{1}}$ to a resolution of $F_{1}$ together with the projection formula, we must have $c_{1}\left(\mathscr{E}_{\mid F_{1}}\right) \equiv 0$ and $c_{2}\left(\mathscr{E}_{\mid F_{1}}\right) \equiv 0$. But then [Ful98, Remark 3.2.4] gives $\mathscr{O}_{\mathbb{P}_{F_{1}}\left(\mathscr{E}_{\mid F_{1}}\right)}(1)^{r} \equiv 0$, yielding a contradiction. This shows that $q$ is equidimensional. By [AD14, Proposition 4.10], there is a vector bundle $\mathscr{G}$ on $Y$ such that

$$
(Z, \mathscr{L}) \cong\left(\mathbb{P}_{Y}(\mathscr{G}), \mathscr{O}_{\mathbb{P}_{Y}(\mathscr{G})}(1)\right)
$$

But then, we must have $\mathbb{P}_{X}(\mathscr{E}) \cong X \times_{Y} \mathbb{P}_{Y}(\mathscr{G})$. This immediately implies that $\mathscr{E} \cong f^{*} \mathscr{G}$, completing the proof of the theorem.

Proof of Theorem 1.9. Notice that the locally free sheaf $\mathscr{E}$ is $f$-numerically flat by Lemma 2.13 .

We prove Theorem 1.9 by induction on $\operatorname{dim} X-\operatorname{dim} Y$.

If $\operatorname{dim} X=\operatorname{dim} Y$, then Theorem 1.9 follows from [GKPT19, Theorem 5.1]. Suppose from now on that $\operatorname{dim} Y<\operatorname{dim} X$.

Then $X$ is uniruled, and thus, we may run a minimal model program for $X$ and end with a Mori fiber space (see [BCHM10, Corollary 1.3.3]). There exists a sequence of maps

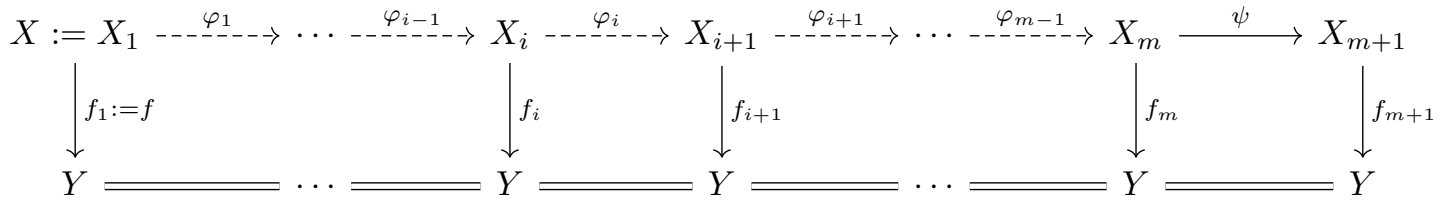

where the $\varphi_{i}$ are either divisorial contractions or flips, and $\psi$ is a Mori fiber space. The spaces $X_{i}$ are normal, $\mathbb{Q}$-factorial, and $X_{i}$ has klt singularities for all $1 \leqslant i \leqslant m$. Moreover, by [KMM87, Lemma 5.1.5], $X_{m+1}$ is also $\mathbb{Q}$-factorial. Applying [Fuj99, Corollary 4.6], we see that $X_{m+1}$ is klt as well.

We construct smooth projective varieties $Z_{i}$ inductively for any integer $1 \leqslant i \leqslant m+1$ as follows. Let $Z_{m+1} \rightarrow X_{m+1}$ be a resolution of $X_{m+1}$, and let $Z_{i}$ be a resolution of the graph of the rational map $X_{i} \rightarrow Z_{i+1}$ for $1 \leqslant i \leqslant m$. We obtain a commutative diagram as follows:

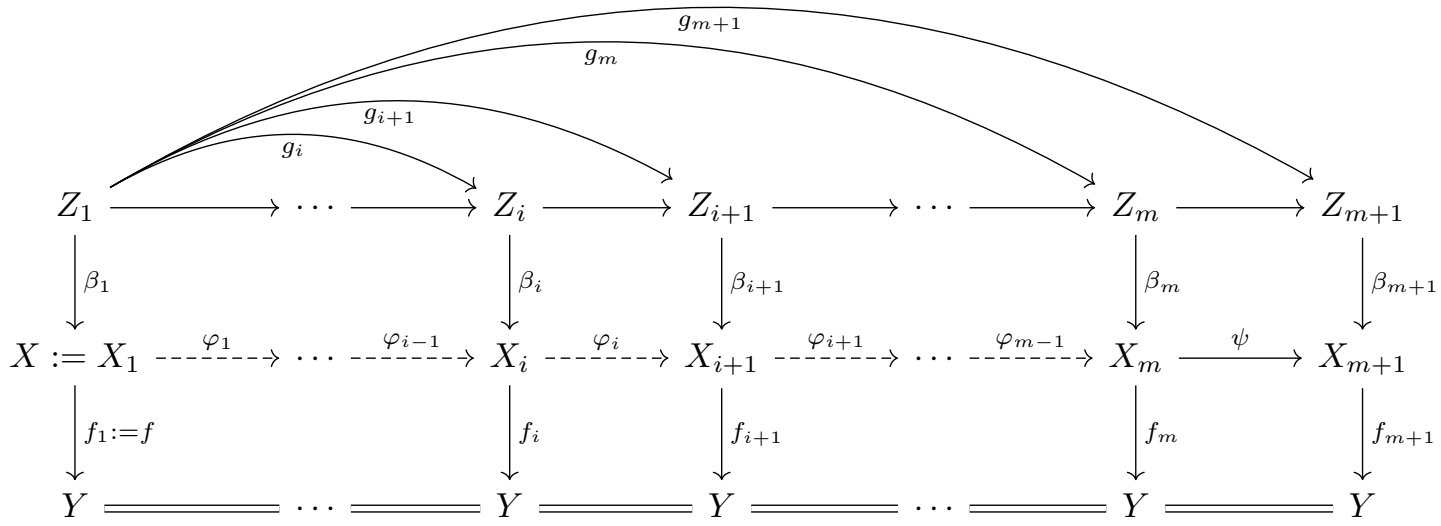

Next, we show inductively that there exist locally free and $f_{i}$-numerically flat sheaves $\mathscr{E}_{i}$ on $X_{i}$ such that $\beta_{1}^{*} \mathscr{E} \cong\left(\beta_{i} \circ g_{i}\right)^{*} \mathscr{E}_{i}$. Set $\mathscr{E} 1:=\mathscr{E}$.

Let $1 \leqslant i \leqslant m-1$. Suppose first that $\varphi_{i}$ is a divisorial contraction. Then Theorem 4.1 shows that there exists a locally free sheaf $\mathscr{E}_{i+1}$ on $X_{i+1}$ such that $\mathscr{E}_{i} \cong \varphi_{i}^{*} \mathscr{E}_{i+1}$. Note that $\mathscr{E}_{i+1}$ is obviously $f_{i+1}$-numerically flat and that $\beta_{1}^{*} \mathscr{E} \cong\left(\beta_{i+1} \circ g_{i+1}\right)^{*} \mathscr{E}_{i+1}$.

Suppose now that $\varphi_{i}$ is the flip of a small extremal contraction $c_{i}: X_{i} \rightarrow Y_{i}$ over $Y$, and let $c_{i+1}: X_{i+1} \rightarrow Y_{i}$ be the natural $Y$-morphism. By Theorem 4.1, there exists a locally free sheaf $\mathscr{G}_{i}$ on $Y_{i}$ such that $\mathscr{E}_{i} \cong c_{i}^{*} \mathscr{G}_{i}$. Set $\mathscr{E}_{i+1}:=c_{i+1}^{*} \mathscr{G}_{i}$. Then $\mathscr{E}_{i+1}$ is $f_{i+1}$-numerically flat and $\beta_{1}^{*} \mathscr{E} \cong\left(\beta_{i+1} \circ g_{i+1}\right)^{*} \mathscr{E}_{i+1}$.

If $i=m$, then Theorem 4.1 again shows that there exists a locally free sheaf $\mathscr{E}_{m+1}$ on $X_{m+1}$ such that $\mathscr{E}_{m} \cong \psi^{*} \mathscr{E}_{m+1}$. The sheaf $\mathscr{E}_{m+1}$ is $f_{m+1}$-numerically flat and $\beta_{1}^{*} \mathscr{E} \cong\left(\beta_{m+1} \circ g_{m+1}\right)^{*} \mathscr{E}_{m+1}$.

Now, a general fiber of $f$ is rationally chain connected by assumption and hence rationally connected since it is smooth. This easily implies that the general fibers of $f_{m+1} \circ \beta_{m+1}: Z_{m+1} \rightarrow Y$ are rationally connected. The 
induction hypothesis applied to $\beta_{m+1}^{*} \mathscr{E}_{m+1}$ then implies that there exists a locally free sheaf $\mathscr{G}$ on $Y$ such that $\beta_{m+1}^{*} \mathscr{E}_{m+1} \cong\left(f_{m+1} \circ \beta_{m+1}\right)^{*} \mathscr{G}$. One readily checks that $\mathscr{E} \cong f^{*} \mathscr{G}$, completing the proof of the corollary.

\section{Preparation For the proof of the main Results}

In the section we provide a technical tool for the proof of Theorem 1.1. We will prove Theorem 1.1 by induction on the dimension. The following result will be useful for the induction process.

Proposition 5.1. Let $X$ be a normal projective variety, and let $D$ be a reduced effective divisor on $X$ such that $(X, D)$ is $\log$ canonical and $K_{X}+D \sim_{\mathbb{Q}} 0$. Suppose that $X$ is $\mathbb{Q}$-factorial with klt singularities. Suppose in addition that there exist a locally free, R-flat sheaf $\mathscr{E}$ on $X$ and an inclusion $\Omega_{X}^{[1]}(\log D) \subseteq \mathscr{E}$ with torsion free cokernel. Then there exist normal projective varieties $Y$ and $T$ as well as a finite cover $\gamma: Y \rightarrow X$ and a dominant rational map $a: Y \rightarrow T$ such that the following holds.

(1) The morphism $\gamma$ is quasi-étale over $X \backslash \operatorname{Supp} D$.

(2) The variety $T$ is $\mathbb{Q}$-factorial and klt with $K_{T} \sim_{\mathbb{Z}} 0$.

(3) There exist open sets $T^{\circ} \subseteq T$ and $Y^{\circ} \subseteq Y$ with complement of codimension at least 2 such that the map a restricts to a projective morphism with rationally chain connected fibers $a^{\circ}: Y^{\circ} \rightarrow T^{\circ}$. Moreover, there is no a-exceptional divisor on $Y$.

(4) There exist a locally free, $R$-flat sheaf $\mathscr{G}$ on $T$ and an inclusion $\Omega_{T}^{[1]} \subseteq \mathscr{G}$ with torsion free cokernel such that $\gamma^{*} \mathscr{E}_{\mid Y^{\circ}} \cong\left(a^{\circ}\right)^{*} \mathscr{G}_{\mid T^{\circ}}$.

We will need the following easy observation.

Lemma 5.2. Let $X$ be a normal variety and let $\mathscr{E}$ be a locally free sheaf on $X$. Let $\mathscr{G} \subseteq \mathscr{E}$ be a reflexive subsheaf. If the quotient sheaf $\mathscr{E} / \mathscr{G}$ is torsion free in codimension 1 , then $\mathscr{G}$ is saturated in $\mathscr{E}$.

Proof. By [Har80, Proposition 1.1], the saturation $\mathscr{G}_{1}$ of $\mathscr{G}$ in $\mathscr{E}$ is reflexive. On the other hand, by assumption, $\mathscr{G}$ and $\mathscr{G}_{1}$ agree in codimension 1 . But this immediately implies that $\mathscr{G}=\mathscr{G}_{1} \subseteq \mathscr{E}$ (see [Har80, Proposition $1.6])$.

Before we give the proof of Proposition 5.1, we need the following auxiliary results.

Lemma 5.3. Let $X$ be a normal projective variety, and let $D$ be a reduced effective divisor on $X$. Suppose that $X$ is $\mathbb{Q}$-factorial with klt singularities. Let $\varphi: X \rightarrow X_{1}$ be a divisorial Mori contraction with exceptional divisor $E$. Suppose furthermore that there exist a locally free, $R$-flat sheaf $\mathscr{E}$ on $X$ and an inclusion $\Omega_{X}^{[1]}(\log D) \subseteq \mathscr{E}$ with torsion free cokernel. Then $E$ is contained in the support of $D$.

Proof. Recall that $E$ is an irreducible divisor since $X$ is $\mathbb{Q}$-factorial. We argue by contradiction and assume that $E$ is not contained in the support of $D$. By Theorem 4.1, there exists a locally free sheaf $\mathscr{E} 1$ on $X_{1}$ such that $\mathscr{E} \cong \varphi^{*} \mathscr{E} 1$. Let $\beta: Y \rightarrow X$ be a canonical resolution of $(X, D)$ and let $B$ be the largest reduced divisor contained in $\beta^{-1}(\operatorname{Supp} D)$. Let $F$ be the strict transform of $E$ in $Y$. By [BCHM10, Lemma 3.6.2], $F$ is covered by curves $C$ contracted by $\varphi \circ \beta$ such that $F \cdot C<0$. Suppose that $C \not \subset \operatorname{Supp} B$. Then the composed map of locally free sheaves

$$
\beta^{*} \mathscr{E}^{*}{ }_{\mid C} \rightarrow \beta^{*} T_{X}(-\log D)_{\mid C} \cong \beta^{*}\left(\beta_{*} T_{Y}(-\log B)\right)_{\mid C} \rightarrow T_{Y}(-\log B)_{\mid C} \rightarrow \mathscr{N}_{F / Y}{ }_{\mid C} \cong \mathscr{O}_{Y}(-F)_{\mid C}
$$

is generically surjective. But $\beta^{*} \mathscr{E}^{*} \mid C$ is the trivial vector bundle while $F \cdot C<0$ by choice of $C$. This yields a contradiction and shows that $E$ is contained in the support of $D$, completing the proof of the lemma.

Lemma 5.4. Let $X$ be a normal projective variety, and let $D$ be a reduced effective divisor on $X$ such that $(X, D)$ is $\log$ canonical and $K_{X}+D \sim_{\mathbb{Q}} 0$. Suppose that $X$ is $\mathbb{Q}$-factorial with klt singularities. Suppose in addition that there exist a locally free, $R$-flat sheaf $\mathscr{E}$ on $X$ and an inclusion $\Omega_{X}^{[1]}(\log D) \subseteq \mathscr{E}$ with torsion free cokernel. Let $\psi: X \rightarrow Y$ be a Mori fiber space. Write $D=R+S$ where any irreducible component of $R$ (resp. $S$ ) maps onto $Y$ (resp. a proper subset of $Y$ ) and let $B$ be the $\mathbb{Q}$-divisor on $Y$ such that $S=\psi^{*} B$. Then there exists a finite cover $\tau: Y_{1} \rightarrow Y$ such that the following holds. Let $X_{1}$ be the normalization of the product $Y_{1} \times_{Y} X$ with natural morphisms $\psi_{1}: X_{1} \rightarrow Y_{1}$ and $\tau_{1}: X_{1} \rightarrow X$. Set $D_{1}:=\tau_{1}^{*}\left(K_{X}+D\right)-K_{X_{1}}$.

(1) The morphism $\tau_{1}$ is quasi-étale.

(2) Set $B_{1}:=\left\ulcorner\tau^{*} B\right\urcorner$. Then $B_{1}$ is the discriminant divisor of $\left(\psi_{1}, D_{1}\right)$. Moreover, $B_{1}$ is reduced and $K_{Y_{1}}+B_{1} \sim \mathbb{Q} 0$.

(3) The pair $\left(Y_{1}, B_{1}\right)$ is log canonical and $Y_{1}$ is of klt type. 
(4) There exists a locally free, $R$-flat sheaf $\mathscr{G}_{1}$ on $Y_{1}$ such that $\psi_{1}^{*} \mathscr{G}_{1} \cong \tau_{1}^{*} \mathscr{E}$, and an inclusion $\Omega_{Y_{1}}^{[1]}\left(\log B_{1}\right) \subseteq$ $\mathscr{G}_{1}$ with torsion free cokernel.

Remark 5.5. The existence of $B$ in the statement of Lemma 5.4 is guaranteed by [Lemma 3.2.5 (2)][KMM87].

Proof of Lemma 5.4. Since $\psi$ is a Mori fiber space and $X$ is $\mathbb{Q}$-factorial by assumption, for any prime divisor $P$ on $Y$, Supp $\psi^{*}(P)$ is irreducible. In particular, for any prime divisor $Q$ on $X, \psi(Q)$ has codimension at most 1. In other words, there is no $\psi$-exceptional divisor on $X$.

By [Zha06, Theorem 1], the general fibers of $\psi$ are rationally connected. This in turn implies that any fiber of $\psi$ is rationally chain connected. Therefore, by Theorem 4.1 applied to $\psi$, there exists a vector bundle $\mathscr{G}$ on $Y$ such that $\mathscr{E} \cong \psi^{*} \mathscr{G}$. Notice that $\mathscr{G}$ is R-flat by Lemma 2.14 .

We first compute the discriminant divisor of $(\psi, D)$. Let $P$ be a prime divisor on $Y$ which is not contained in the support of $B$ and write $\psi^{*} P=m Q$ where $m$ is a positive integer and $Q$ is a prime divisor. Let $Y^{\circ} \subseteq Y_{\text {reg }} \backslash$ Supp $B$ be an open set such that $Y^{\circ} \cap P \neq \emptyset$, and set $X^{\circ}:=\psi^{-1}\left(Y^{\circ}\right)$. We may assume without loss of generality that there exists a finite morphism $\tau^{\circ}: Y_{1}^{\circ} \rightarrow Y^{\circ}$ with $Y_{1}^{\circ}$ smooth such that $\tau^{\circ}$ branches only over $P \cap Y^{\circ}$ with ramification index $m$. Let $X_{1}^{\circ}$ denotes the normalization of the fiber product $Y_{1}^{\circ} \times_{Y} X$ with natural morphisms $\psi_{1}^{\circ}: X_{1}^{\circ} \rightarrow Y_{1}^{\circ}$ and $\tau_{1}^{\circ}: X_{1}^{\circ} \rightarrow \psi^{-1}\left(Y^{\circ}\right)=: X^{\circ}$. Then $\tau_{1}^{\circ}$ is a quasi-étale morphism. Moreover, shrinking $Y^{\circ}$, if necessary, we may assume that $\psi_{1}^{\circ}$ has reduced fibers. Set $D_{1}^{\circ}:=\left(\tau_{1}^{\circ}\right)^{*} D_{\mid X^{\circ},}, \mathscr{E}_{1}^{\circ}:=\left(\tau_{1}^{\circ}\right)^{*} \mathscr{E} \mid X^{\circ}$ and $\mathscr{G}_{1}^{\circ}:=\left(\tau^{\circ}\right)^{*} \mathscr{G}_{\mid X^{\circ}}$. Observe now (see Lemma 2.7) that

$$
\Omega_{X_{1}^{\circ}}^{[1]}\left(\log D_{1}^{\circ}\right) \cong\left(\tau_{1}^{\circ}\right)^{*} \Omega_{X^{\circ}}^{[1]}\left(\log D_{\mid X^{\circ}}\right) .
$$

By Lemma 5.2, we have an inclusion $\Omega_{X_{1}^{\circ}}^{[1]}\left(\log D_{1}^{\circ}\right) \subseteq \mathscr{E}_{1}^{\circ}$ with torsion free cokernel. Notice that any irreducible component of $D_{1}^{\circ}$ dominates $Y_{1}^{\circ}$ by construction. Therefore, shrinking $Y^{\circ}$ again, if necessary, we may assume that the locus where the composed map

$$
\left(\psi_{1}^{\circ}\right)^{*}\left(\mathscr{G}_{1}^{\circ}\right)^{*} \cong\left(\mathscr{E}_{1}^{\circ}\right)^{*} \rightarrow T_{X_{1}^{\circ}}\left(-\log D_{1}^{\circ}\right) \rightarrow T_{X_{1}^{\circ}} \rightarrow\left(\psi_{1}^{\circ}\right)^{*} T_{Y_{1}^{\circ}}
$$

is not surjective does not contain any fiber of $\psi_{1}$. This easily implies that the induced map of locally free sheaves $\left(\mathscr{E}_{1}^{\circ}\right)^{*} \rightarrow T_{Y_{1}^{\circ}}$ is surjective. Shrinking $Y^{\circ}$ further, we may assume that $\mathscr{E} \circ$ is trivial and that $\left(\psi_{1}^{\circ}\right)^{*} T_{Y_{1}^{\circ}}$ is a direct summand of $T_{X_{1}^{\circ}}\left(-\log D_{1}^{\circ}\right)$. A classical result of complex analysis says that complex flows of vector fields on analytic spaces exist. This implies that the fibration $\left(X_{1}^{\circ}, D_{1}^{\circ}\right) \rightarrow Y_{1}^{\circ}$ is locally trivial for the analytic topology. One then readily checks that the discriminant divisor $B_{Y}$ of $(\psi, D)$ is

$$
B_{Y}=\ulcorner B\urcorner+\sum \frac{m_{P}-1}{m_{P}} P
$$

where $P$ runs through all prime divisors on $Y$ not contained in the support of $B$, and $m_{P}$ denotes the multiplicity of $\psi^{*} P$ along $\operatorname{Supp} \psi^{*}(P)$.

By Corollary 3.6 applied to $\psi$, we must have $K_{Y}+B_{Y} \sim_{\mathbb{Q}} 0$. Let $\tau: Y_{1} \rightarrow Y$ be the corresponding index one cover (see [Sho92, Section 2.4]). Let $X_{1}$ be the normalization of the product $Y_{1} \times_{Y} X$ with natural morphisms $\psi_{1}: X_{1} \rightarrow Y_{1}$ and $\tau_{1}: X_{1} \rightarrow X$. By construction, $\tau$ is étale at the generic points of Supp $B$. Moreover, if $P$ is a prime divisor on $Y$ which is not contained in the support of $B$, then $\tau$ has ramification index $m_{P}$ along any irreducible component of $\tau^{-1}(P)$. This easily implies that $\tau_{1}$ is quasi-étale. Set $\mathscr{E}_{1}:=\tau_{1}^{*} \mathscr{E}$ and $D_{1}:=\tau_{1}^{*} D$. Notice that $\mathscr{E}_{1}$ is R-flat and that we have an inclusion $\Omega_{X_{1}}^{[1]}\left(\log D_{1}\right) \cong \tau^{[*]} \Omega_{X}^{[1]}(\log D) \subseteq \mathscr{E}_{1}=\tau_{1}^{*} \mathscr{E}$ with torsion free cokernel (see Lemma 5.2). Notice also that the fibration $\left(\psi_{1}, D_{1}\right)$ is generically isotrivial. Moreover, arguing as in the previous paragraph, we see that its discriminant is the reduced divisor $B_{1}=\left\ulcorner\tau^{*} B\right\urcorner$. By construction, we have $K_{Y_{1}}+B_{1} \sim_{\mathbb{Q}} 0$. This proves Items (1) and (2).

To prove Item (3), recall that $B_{1}$ is the discriminant of $\left(\psi_{1}, D_{1}\right)$. Set $D_{i}:=\left(1-\frac{1}{i}\right) D$ and $D_{1, i}:=\tau_{1}^{*} D_{i}$ for any integer $i \geqslant 1$. Notice that $D_{1, i}$ is effective. Observe also that $\left(X, D_{i}\right)$ is klt for $i \geqslant 1$ since $X$ is $\mathbb{Q}$-factorial and klt and $(X, D)$ is $\log$ canonical by assumption. It follows that $\left(X_{1}, D_{1, i}\right)$ is klt. Let $B_{i}$ (resp. $\left.B_{1, i}\right)$ be the discriminant divisor of $\left(\psi, D_{i}\right)$ (resp. $\left.\left(\psi_{1}, D_{1, i}\right)\right)$. The $\mathbb{Q}$-divisor $K_{Y}+B_{i}$ is $\mathbb{Q}$-Cartier since $Y$ is $\mathbb{Q}$-factorial by [KMM87, Lemma 5.1.5]. By Fact 2.17, we have $K_{Y_{1}}+B_{1, i} \sim_{\mathbb{Q}} \tau^{*}\left(K_{Y}+B_{i}\right)$ so that $K_{Y_{1}}+B_{1, i}$ is $\mathbb{Q}$-Cartier as well. On the other hand, since $K_{X}+D \equiv 0$ and $-K_{X}$ is $\psi$-ample, we see that $-\left(K_{X_{1}}+D_{1, i}\right)$ is $\psi_{1}$-ample. By Lemma 2.18 applied to $\left(\psi_{1}, D_{1, i}\right)$, we conclude that $\left(Y_{1}, B_{1, i}\right)$ is klt. Clearly, $B_{1, i} \leqslant B_{1}$ and $B_{1, i} \rightarrow B_{1}$ as $i \rightarrow+\infty$. This proves Item (3). 
Set $\mathscr{G}_{1}:=\tau^{*} \mathscr{G}$. Note that $\mathscr{G}_{1}$ is R-flat. By Lemma 2.8 , the standard pull-back map of Kähler differential induces an injective map

$$
\Omega_{Y_{1}}^{[1]}\left(\log B_{1}\right) \rightarrow\left(\left(\psi_{1}\right)_{*} \Omega_{X_{1}}^{[1]}\left(\log D_{1}\right)\right)^{* *} \cong \mathscr{G}_{1}
$$

By construction, $\psi_{1}$ has reduced fibers on some open set in $Y_{1} \backslash \operatorname{Supp} B_{1}$ with complement of codimension at least 2. An easy local computation (see [Del70, Chapitre 2, Proposition 3.2]) then shows that the cokernel of the inclusion $\Omega_{Y_{1}}^{[1]}\left(\log B_{1}\right) \subset \mathscr{G}_{1}$ is torsion free in codimension 1 , and hence torsion free by Lemma 5.2. This finishes the proof of the lemma

We are now in position to prove Proposition 5.1.

Proof of Proposition 5.1. We prove Proposition 5.1 by induction on $\operatorname{dim} X$.

If $\operatorname{dim} X=1$, then either $(X, D) \cong\left(\mathbb{P}^{1},[0]+[\infty]\right)$, or $X$ is a Riemann surface of genus 1 and $D=0$. So the statement holds true in this case.

Suppose from now on that $\operatorname{dim} X \geqslant 2$.

If $D=0$, then $K_{X}$ is torsion by assumption. Let $X_{1} \rightarrow X$ be the corresponding index one cover, which is quasi-étale ([KM98, Lemma 2.53]). Then $X_{1}$ is klt and $K_{X_{1}} \sim_{\mathbb{Z}} 0$ by construction. So the statement holds true in this case.

Suppose from now on that $D \neq 0$. Then $X$ is uniruled by [MM86] applied to a resolution of $(X, D)$. Thus, we may run a minimal model program for $X$ and end with a Mori fiber space (see [BCHM10, Corollary 1.3.3]). There exists a sequence of maps

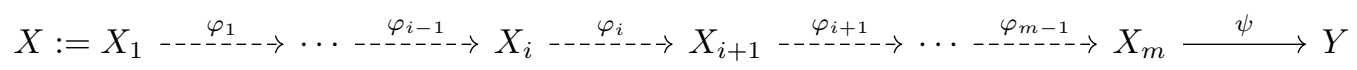

where the $\varphi_{i}$ are either divisorial contractions or flips, and $\psi$ is a Mori fiber space. The spaces $X_{i}$ are normal, $\mathbb{Q}$-factorial, and $X_{i}$ has klt singularities for all $1 \leqslant i \leqslant m$. Let $D_{i}$ be the push-forward of $D$ on $X_{i}$. By [KMM87, Lemma 3.2.5 (2)], we have $K_{X_{i}}+D_{i} \sim_{\mathbb{Q}} 0$. Moreover, the pair $\left(X_{i}, D_{i}\right)$ is log canonical by [KM98, Lemma 3.38].

We define projective varieties $W_{i}$ inductively for any integer $1 \leqslant i \leqslant m$ as follows. Set $W_{m}:=X_{m}$. Let $W_{i}$ be a resolution of the graph of the rational map $X_{i} \rightarrow W_{i+1}$ for $1 \leqslant i \leqslant m-1$ with morphism $p_{i}: W_{i} \rightarrow X_{i}$. Let $q_{i}: W_{1} \rightarrow W_{i}$ be the natural morphism. We then show inductively that there exist locally free, R-flat sheaves $\mathscr{E}_{i}$ on $X_{i}$ and an inclusion $\Omega_{X_{i}}^{[1]}\left(\log D_{i}\right) \subseteq \mathscr{E}_{i}$ with torsion free cokernel such that $p_{1}^{*} \mathscr{E} \cong\left(p_{i} \circ q_{i}\right)^{*} \mathscr{E}_{i}$. Set $\mathscr{E}_{1}:=\mathscr{E}$.

Suppose that $\varphi_{i}$ is a divisorial contraction. By Theorem 4.1, there exists a locally free sheaf $\mathscr{E}_{i+1}$ on $X_{i+1}$ such that $\mathscr{E}_{i} \cong \varphi_{i}^{*} \mathscr{E}_{i+1}$. Moreover, $\mathscr{E}_{i+1}$ is R-flat by Lemma 2.14 .

Suppose now that $\varphi_{i}$ is the flip of a small extremal contraction $c_{i}: X_{i} \rightarrow Z_{i}$, and let $c_{i+1}: X_{i+1} \rightarrow Z_{i}$ be the natural morphism. By Theorem 4.1 again, there exists a locally free sheaf $\mathscr{G}_{i}$ on $Z_{i}$ such that $\mathscr{E}_{i} \cong c_{i}^{*} \mathscr{G}_{i}$. Set $\mathscr{E}_{i+1}:=c_{i+1}^{*} \mathscr{G}_{i}$. Then $\mathscr{G}_{i}$ is R-flat by Lemma 2.14 and hence so is $\mathscr{E}_{i+1}$.

In either case, the inclusion $\Omega_{X_{i}}^{[1]}\left(\log D_{i}\right) \subseteq \mathscr{E}_{i}$ induces an inclusion $\Omega_{X_{i+1}}^{[1]}\left(\log D_{i+1}\right) \subseteq \mathscr{E}_{i+1}$ with torsion free cokernel by Lemma 5.2. Moreover, one readily checks that $p_{1}^{*} \mathscr{E} \cong\left(p_{i+1} \circ q_{i+1}\right)^{*} \mathscr{E}_{i+1}$.

Then, we apply Lemma 5.4 to $\psi$. Write $D_{m}=R+S$ where any irreducible component of $R$ (resp. $S$ ) maps onto $Y$ (resp. a proper subset of $Y$ ) and let $B$ be the $\mathbb{Q}$-divisor on $Y$ such that $S=\psi^{*} B$ (see [KMM87, Lemma 3.2.5 (2)]). There exists a finite cover $\tau: Y_{1} \rightarrow Y$ such that the following holds. Let $X_{m+1}$ be the normalization of the product $Y_{1} \times_{Y} X_{m}$ with natural morphisms $\psi_{1}: X_{m+1} \rightarrow Y_{1}$ and $\tau_{1}: X_{m+1} \rightarrow X_{m}$.

- The morphism $\tau_{1}$ is quasi-étale.

- The divisor $B_{1}:=\left\ulcorner\tau^{*} B\right\urcorner$ is reduced and $K_{Y_{1}}+B_{1} \sim_{\mathbb{Q}} 0$.

- The pair $\left(Y_{1}, B_{1}\right)$ is $\log$ canonical and $Y_{1}$ is of klt type.

- There exists a locally free, R-flat sheaf $\mathscr{G}_{1}$ on $Y_{1}$ such that $\psi_{1}^{*} \mathscr{G}_{1} \cong \tau_{1}^{*} \mathscr{E}$, and an inclusion $\Omega_{Y_{1}}^{[1]}\left(\log B_{1}\right) \subseteq \mathscr{G}_{1}$ with torsion free cokernel.

Let $\beta: Y_{2} \rightarrow Y_{1}$ be a $\mathbb{Q}$-factorialization of $Y_{1}$, whose existence is established in [Kol13, Corollary 1.37]. Recall that $\beta$ is a small birational projective morphism and that $Y_{2}$ is $\mathbb{Q}$-factorial with klt singularities. Set $B_{2}:=\left(\beta^{-1}\right)_{*} B_{1}$. Then $\beta^{*} \mathscr{G}_{1}$ is obviously R-flat and there is an inclusion $\Omega_{Y_{2}}^{[1]}\left(\log B_{2}\right) \subseteq \beta^{*} \mathscr{G}_{1}$ with torsion free cokernel (see Lemma 5.2). Moreover, $\left(Y_{2}, B_{2}\right)$ is log canonical by [Kol97, Lemma 3.10] and $K_{Y_{2}}+B_{2} \sim_{\mathbb{Q}} 0$.

The induction hypothesis applied to $\left(Y_{2}, B_{2}\right)$ asserts that there exist normal projective varieties $Z_{2}$ and $T$ as well as a finite cover $\eta: Z_{2} \rightarrow Y_{2}$ and a dominant rational map $a: Z_{2} \rightarrow T$ such that the following holds. 
- The morphism $\eta$ is quasi-étale over $Y_{2} \backslash \operatorname{Supp} B_{2}$.

- The variety $T$ is $\mathbb{Q}$-factorial and klt with $K_{T} \sim_{\mathbb{Z}} 0$.

- There exist open sets $T^{\circ} \subseteq T$ and $Z_{2}^{\circ} \subseteq Z_{2}$ with complement of codimension at least 2 such that the map $a$ restricts to a projective morphism with rationally chain connected fibers $a^{\circ}: Z_{2}^{\circ} \rightarrow T^{\circ}$. Moreover, there is no $a$-exceptional divisor on $Z_{2}$.

- There exist a locally free, R-flat sheaf $\mathscr{G}$ on $T$ and an inclusion $\Omega_{T}^{[1]} \subseteq \mathscr{G}$ with torsion free cokernel such that $(\eta \circ \beta)^{*} \mathscr{G}_{1_{\mid Z}^{\circ}} \cong\left(a^{\circ}\right)^{*} \mathscr{G}_{\mid T^{\circ}}$.

Let $Z_{1}$ be the normalization of the product $X_{m+1} \times_{Y_{1}} Z_{2}$ and let $Z$ be the normalization of $X_{1}$ in the function field of $Z_{1}$. We have a diagram as follows:

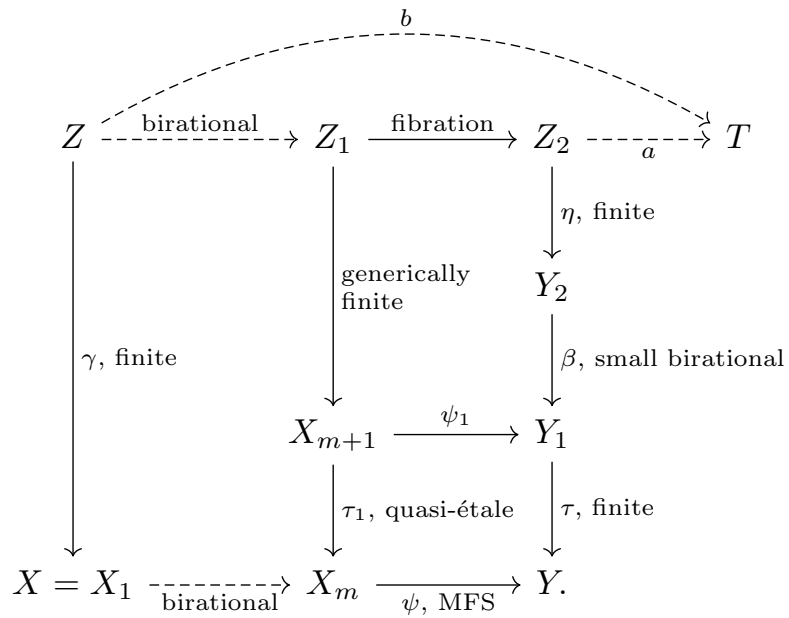

We now show that $\gamma$ is quasi-étale over $X \backslash \operatorname{Supp} D$. Let $P$ be a prime divisor on $X$ and suppose that $\gamma$ is ramified over $P$. If $P$ is contracted by the birational map $X \rightarrow X_{m}$ then $P$ is contained in the support of $D$ by Lemma 5.3. Suppose that $P$ is not contracted by $X \rightarrow X_{m}$ and let $P_{m}$ be the push-forward of $P$ on $X_{m}$. Then the generically finite map $Z_{1} \rightarrow X_{m+1}$ is ramified over $\tau_{1}^{*} P_{m}$. On the other hand, since $\psi$ is a Mori fiber space, any irreducible component of $\tau_{1}^{*} P_{m}$ maps onto a closed subset of codimension at most 1 in $Y_{1}$. Thus, since $\eta$ is quasi-étale over $Y_{2} \backslash \operatorname{Supp} B_{2}$ and $\beta$ is a small birational contraction, any irreducible component of $\tau_{1}^{*} P_{m}$ must be contained in the support of $\psi_{1}^{*} B_{1}$. This shows that $P$ is contained in the support of $D$.

Notice that $T$ has canonical singularities since $K_{T} \sim_{\mathbb{Z}} 0$. This implies that $T$ is not uniruled. By assumption, $X$ is $\mathbb{Q}$-factorial with klt singularities and $(X, D)$ is $\log$ canonical. Applying Lemma 2.6, we see that $Z$ is of klt type. This in turn implies that the rational map $b: Z \rightarrow T$ is almost proper by [HM07, Corollary 1.7]. By [HM07, Corolary 1.8] together with [HM07, Theorem 1.2], the general fibers of $\psi: X_{m} \rightarrow Y$ are rationally connected. This implies that the general fibers of the rational map $Z \rightarrow Z_{2}$ are rationally connected as well. Since the general fibers of $a$ are also rationally connected by [HM07, Corolary 1.8], applying [GHS03, Corollary 1.3], we see that the general fibers of $b: Z \rightarrow T$ are rationally connected. Therefore, $b: Z \rightarrow T$ is the maximally rationally chain connected fibration of $Z$.

Set $G:=\gamma^{*}\left(K_{X}+D\right)-K_{Z}$. By Lemma 2.6, $G$ is effective since $\gamma$ is quasi-étale over $X \backslash \operatorname{Supp} D$. Moreover, the pair $(Z, G)$ is $\log$ canonical, and $\gamma^{-1}(\operatorname{Supp} D) \subseteq \operatorname{Supp} G$.

Let $Q$ be a prime divisor on $Z$. Suppose that $Q$ is $b$-exceptional. If $Q$ is contracted by the rational map $Z \rightarrow X_{m}$ then $Q$ must be contained in the support of $G$ by Lemma 5.3. On the other hand, by Proposition 5.6 below, any irreducible component of $G$ dominates $T$, yielding a contradiction. Therefore, $Q$ is not contracted by the rational map $Z \rightarrow X_{m}$. Then, since $\psi$ is a Mori fiber space, $Q$ is not exceptional for $Z \rightarrow Z_{2}$. It follows that the image of $Q$ on $Z_{2}$ is $a$-exceptional. But this contradicts the induction hypothesis. This shows that there is no $b$-exceptional divisor $Z$.

Suppose now that there is a uniruled prime divisor $P$ on $T$. Set $k:=\operatorname{dim} T$. The inclusion $\Omega_{T}^{[1]} \subseteq \mathscr{G}$ then yields an inclusion

$$
s: \mathscr{O}_{T}\left(K_{T}\right) \cong \mathscr{O}_{T} \subseteq \wedge^{k} \mathscr{G}
$$

with torsion free cokernel at a general point of $P$. Let $\nu: \mathbb{P}^{1} \rightarrow X$ be a rational curve passing through a general point $p \in P$. By general choice of $\nu$, we may assume that $s(p) \neq 0$. On the other hand, $\nu^{*}\left(\wedge^{k} \mathscr{G}\right)$ is numerically flat by assumption. This immediately implies that $s$ is nowhere vanishing along $\nu\left(\mathbb{P}^{1}\right)$. By [DPS94, Lemma 
1.20], the reflexive sheaf $\Omega_{T}^{[1]}$ is then locally free in a neighbourhood of $\nu\left(\mathbb{P}^{1}\right)$. This in turn implies that $T$ is smooth along $\nu\left(\mathbb{P}^{1}\right)$ by the solution of the Zariski-Lipman conjecture for log canonical spaces (see [Dru14, Theorem 1.1] or [GK14, Corollary 1.3]). Then $\nu^{*} T_{T} \cong \mathscr{O}_{\mathbb{P}^{1}}^{\oplus k}$, yielding a contradiction since $T_{\mathbb{P}^{1}} \subset \nu^{*} T_{T}$. By [HM07, Corollary 1.7], there exist open sets $T^{\circ} \subseteq T$ and $Z^{\circ} \subseteq Z$ such that $b$ restricts to a projective morphism with connected fibers $b^{\circ}: Z^{\circ} \rightarrow T^{\circ}$ and such that $T^{\circ}$ has complement of codimension at least 2 . Since there is no $b$-exceptional divisor on $Z, Z^{\circ}$ has complement of codimension at least 2 .

Finally, one readily checks that $\left(b^{\circ}\right)^{*} \mathscr{G}_{T^{\circ}} \cong f^{*} \mathscr{E}_{\mid Z^{\circ}}$, finishing the proof of the proposition.

Proposition 5.6 ([Zha05, Main Theorem and Remark 1]). Let $(X, D)$ be a log canonical pair with X projective and $D$ effective, and let $f: X \rightarrow T$ be the maximally rationally chain connected fibration. Suppose that $-\left(K_{X}+D\right)$ is nef. Then $f$ is semistable in codimension 1 . Moreover, any irreducible component of $D$ dominates $T$.

\section{Proofs}

In this section we prove our main results. Note that Theorem 1.1 is an immediate consequence of Theorem 6.1 below.

Theorem 6.1. Let $X$ be a normal projective variety of klt type, and let $D$ be a reduced effective divisor on $X$ such that $(X, D)$ is $\log$ canonical. Suppose that the sheaf $\Omega_{X}^{[1]}(\log D)$ is locally free and $R$-flat, and that $-\left(K_{X}+D\right)$ is nef. Then there exist a smooth projective variety $T$ with $K_{T} \equiv 0$ as well as a surjective morphism with connected fibers $a: X \rightarrow T$. The fibration $(X, D) \rightarrow T$ is locally trivial for the analytic topology and any fiber $F$ of the map a is a toric variety with boundary divisor $D_{\mid F}$. Moreover, $T$ contains no rational curve.

Proof. For the reader's convenience, the proof is subdivided into a number of steps.

Step 1. By the cone theorem for $\log$ canonical spaces (see [Fuj11, Theorem 1.4]), we must have $K_{X}+D \equiv 0$ since $-\left(K_{X}+D\right)$ is nef and $\left(K_{X}+D\right) \cdot C=0$ for any rational curve $C \subset X$ by assumption.

Let $\beta: X_{1} \rightarrow X$ be a canonical resolution of $(X, D)$ and let $D_{1}$ be the largest reduced divisor contained in $\beta^{-1}(\operatorname{Supp} D)$. By Lemma 2.10, we have $\Omega_{X_{1}}^{1}\left(\log D_{1}\right) \cong \beta^{*} \Omega_{X}^{1}(\log D)$. In particular, $\Omega_{X_{1}}^{1}\left(\log D_{1}\right)$ is locally free and R-flat. Moreover, $\left(X_{1}, D_{1}\right)$ is $\log$ canonical and $K_{X_{1}}+D_{1} \equiv 0$. Applying [CKP12, Theorem 0.1], we see that $K_{X_{1}}+D_{1}$ is torsion.

Suppose now that the conclusion of Theorem 6.1 holds for the pair $\left(X_{1}, D_{1}\right)$. We show that the conclusion of Theorem 6.1 also holds for the pair $(X, D)$. By assumption, there exist a smooth projective variety $T_{1}$ with $K_{T_{1}} \equiv 0$ as well as a surjective morphism with connected fibers $a_{1}: X_{1} \rightarrow T_{1}$. The fibration $\left(X_{1}, D_{1}\right) \rightarrow T_{1}$ is locally trivial for the analytic topology and any fiber $F_{1}$ of the map $a_{1}$ is a smooth toric variety with boundary divisor $D_{1 \mid F_{1}}$. Moreover, $T_{1}$ contains no rational curve. Observe also that any irreducible component of $D_{1}$ maps onto $T_{1}$.

By [HM07, Theorem 1.2], every fiber of $\beta$ is rationally chain connected. On the other hand, $T_{1}$ contains no rational curve by assumption. It follows that the rational map $a_{1} \circ \beta^{-1}$ is a morphism $a: X \rightarrow T_{1}=: T$. By Theorem 1.9 applied to $a_{1}$ together with the projection formula, there exists a vector bundle $\mathscr{E}$ on $T$ such that $\Omega_{X}^{[1]}(\log D) \cong a^{*} \mathscr{E}$. Since $\left(X_{1}, D_{1}\right)$ is locally trivial over $T_{1}$ (for the analytic topology), the morphism $\mathscr{E}^{*} \rightarrow T_{T}=T_{T_{1}}$ induced by the composed map

$$
a_{1}^{*} \mathscr{E}^{*} \cong T_{X_{1}}\left(-\log D_{1}\right) \rightarrow T_{X_{1}} \rightarrow a_{1}^{*} T_{T_{1}}
$$

is surjective. This easily implies that the composed morphism

$$
a^{*} \mathscr{E}^{*} \cong T_{X}(-\log D) \rightarrow T_{X} \rightarrow a^{*} T_{T}
$$

is surjective as well since $\beta^{*} T_{X}(-\log D) \cong T_{X_{1}}\left(-\log D_{1}\right)$. Moreover, $T_{T}$ is locally a direct summand of $\mathscr{E}^{*}$. Now, a classical result of complex analysis says that complex flows of vector fields on analytic spaces exist. This implies that the fibration $\left(X_{1}, D_{1}\right) \rightarrow T$ is locally trivial for the analytic topology.

Let $F$ be any fiber of $a$ and let $F^{\circ} \subseteq F$ be the open set where $\left(F, D_{\mid F}\right)$ is $\log$ smooth. Note that $\left(F, D_{\mid F}\right)$ is $\log$ canonical. In particular, $F^{\circ}$ has complement of codimension at least 2 in $F$. The sequence

$$
0 \rightarrow T_{F^{\circ}}\left(-\log D_{\mid F^{\circ}}\right) \rightarrow T_{X}(-\log D)_{\mid F^{\circ}} \cong \mathscr{O}_{F^{\circ}}^{\oplus \operatorname{dim} X} \rightarrow \mathscr{N}_{F / X_{\mid F^{\circ}}} \cong \mathscr{O}_{F^{\circ}}^{\oplus \operatorname{dim} T} \rightarrow 0
$$

is exact (see [Dru14, Lemma 3.2]), and hence $T_{F}\left(-\log D_{\mid F}\right) \cong \mathscr{O}_{F}^{\oplus} \operatorname{dim} F$ since both sheaves are reflexive and agree on $F^{\circ}$. Let $\mu: F_{1} \rightarrow F$ be a canonical resolution, and let $D_{F_{1}}$ be the largest reduced divisor contained in 
$\mu^{-1}\left(\operatorname{Supp} D_{\mid F}\right)$. By Lemma 2.10,

$$
\Omega_{F_{1}}^{1}\left(\log D_{F_{1}}\right) \cong \mu^{*} \Omega_{F}^{[1]}\left(\log D_{\mid F}\right) \cong \mathscr{O}_{F_{1}}^{\oplus \operatorname{dim} F_{1}} .
$$

By [Win04, Corollary 1], $F_{1}$ is a toric variety with boundary divisor $D_{F_{1}}$. This in turn implies that $F$ is a toric variety with boundary divisor $D_{\mid F}$. This shows that the conclusion of Theorem 6.1 holds for the pair $(X, D)$. Therefore, we may assume without loss of generality that the following holds.

Assumption 6.2. The pair $(X, D)$ is $\log$ smooth and $K_{X}+D \sim_{\mathbb{Q}} 0$.

We prove Theorem 6.1 by induction on $\operatorname{dim} X$.

If $\operatorname{dim} X=1$, then either $(X, D) \cong\left(\mathbb{P}^{1},[0]+[\infty]\right)$, or $X$ is a Riemann surface of genus 1 and $D=0$. The statement holds true in this case.

Suppose from now on that $\operatorname{dim} X \geqslant 2$ and apply Proposition 5.1. There exist normal projective varieties $X_{1}$ and $T_{1}$ as well as a finite cover $\gamma: X_{1} \rightarrow X$ and a dominant rational map $a_{1}: X_{1} \rightarrow T_{1}$ such that the following properties hold.

- The morphism $\gamma$ is quasi-étale over $X \backslash \operatorname{Supp} D$.

- The variety $T_{1}$ is $\mathbb{Q}$-factorial and klt with $K_{T_{1}} \sim_{\mathbb{Z}} 0$.

- There exist open sets $T_{1}^{\circ} \subseteq T_{1}$ and $X_{1}^{\circ} \subseteq X_{1}$ with complement of codimension at least 2 such that the map $a_{1}$ restricts to a projective morphism with rationally chain connected fibers $a_{1}^{\circ}: X_{1}^{\circ} \rightarrow T_{1}^{\circ}$. Moreover, there is no $a_{1}$-exceptional divisor on $X_{1}$.

- There exist a locally free, R-flat sheaf $\mathscr{G}_{1}$ on $T_{1}$ and an inclusion $\Omega_{T_{1}}^{[1]} \subseteq \mathscr{G}_{1}$ with torsion free cokernel such that $\gamma^{*} \Omega_{X}^{[1]}(\log D)_{\mid X_{1}^{\circ}} \cong\left(a_{1}^{\circ}\right)^{*} \mathscr{G}_{1 \mid T_{1}^{\circ}}$.

Set $D_{1}:=\gamma^{*}\left(K_{X}+D\right)-K_{X_{1}}$ and $D_{1}^{\circ}:=D_{1 \mid X_{1}^{\circ}}$. By Lemma 2.7, we have

$$
\gamma^{*} \Omega_{X}^{[1]}(\log D) \cong \Omega_{X_{1}}^{[1]}\left(\log D_{1}\right) .
$$

Step 2. We show that the fibration $\left(X_{1}^{\circ}, D_{1 \mid X_{1}^{\circ}}\right) \rightarrow T_{1}^{\circ}$ is locally trivial for the analytic topology, and that any fiber $F_{1}$ of $a_{1}^{\circ}$ is a toric variety with boundary divisor $D_{1 \mid F_{1}}$. We may assume without loss of generality that $T_{1}^{\circ}$ is contained in the smooth locus of $T_{1}$. Recall from Proposition 5.6 that any irreducible component of $D_{1}$ maps onto $T_{1}$. By Proposition 5.6 again, we may also assume that $a_{1}$ has reduced fibers over $T_{1}^{\circ}$. It follows that the composed map

$$
\left(a_{1}^{\circ}\right)^{*}\left(\mathscr{G}_{1 \mid T_{1}^{\circ}}^{*}\right) \cong T_{X_{1}^{\circ}}\left(-\log D_{1}^{\circ}\right) \rightarrow T_{X_{1}^{\circ}} \rightarrow\left(a_{1}^{\circ}\right)^{*} T_{T_{1}^{\circ}}
$$

is surjective. Therefore, the induced map $\mathscr{G}_{1 \mid T_{1}^{\circ}} \rightarrow T_{T_{1}^{\circ}}$ is surjective as well, and hence $T_{T_{1}^{\circ}}$ is locally a direct summand of $\mathscr{G}_{1 \mid T_{1}^{*}}^{*}$. As before, this implies that the fibration $\left(X_{1}^{\circ}, D_{1}^{\circ}\right) \rightarrow T_{1}^{\circ}$ is locally trivial for the analytic topology.

Let $F_{1}$ be a general fiber of $a_{1}$, and set $D_{F_{1}}:=D_{1 \mid F_{1}}$. One readily checks that $T_{F_{1}}\left(-\log D_{F_{1}}\right) \cong \mathscr{O}_{F_{1}}^{\oplus \operatorname{dim} F_{1}}$. Let $\mu_{1}: F_{2} \rightarrow F_{1}$ be a canonical resolution, and let $D_{F_{2}}$ be the largest reduced divisor contained in $\mu_{1}^{-1}\left(\operatorname{Supp} D_{F_{1}}\right)$. By Lemma 2.10,

$$
\Omega_{F_{2}}^{1}\left(\log D_{F_{2}}\right) \cong \mu_{1}^{*} \Omega_{F_{1}}^{[1]}\left(\log D_{F_{1}}\right) \cong \mathscr{O}_{F_{2}}^{\oplus \operatorname{dim} F_{2}} .
$$

It follows that $F_{2}$ is a toric variety with boundary divisor $D_{F_{2}}$ (see [Win04, Corollary 1]). This in turn implies that $F_{1}$ is a toric variety with boundary divisor $D_{F_{1}}$, completing the proof of the claim.

Step 3. Let $\beta_{1}: X_{2} \rightarrow X_{1}$ be a canonical resolution of $\left(X_{1}, D_{1}\right)$ and let $D_{2}$ be the largest reduced divisor contained in $\beta_{1}^{-1}\left(\operatorname{Supp} D_{1}\right)$. By Lemma 2.10, we have $\Omega_{X_{2}}^{1}\left(\log D_{2}\right) \cong \beta^{*} \Omega_{X_{1}}^{1}\left(\log D_{1}\right)$. In particular, $\Omega_{X_{2}}^{1}\left(\log D_{2}\right)$ is locally free and R-flat. Moreover, $\left(X_{2}, D_{2}\right)$ is $\log$ canonical and $K_{X_{2}}+D_{2} \sim_{\mathbb{Q}} 0$. Set $a_{2}:=a_{1} \circ \beta_{1}^{-1}: X_{2} \rightarrow T_{1}$. We have a commutative diagram as follows:

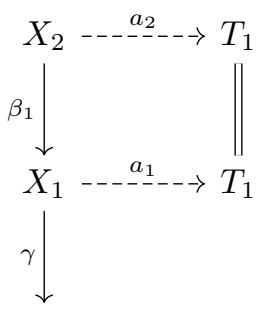

$X$. 
By Proposition 5.6, any irreducible component of $D_{2}$ maps onto $T_{1}$. In particular, there is no $a_{2}$-exceptional

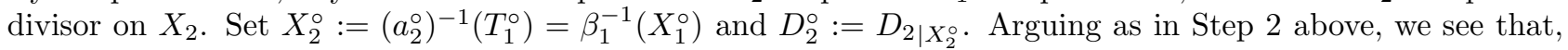
shrinking $T_{1}^{\circ}$ if necessary, we may assume that the fibration $\left(X_{2}^{\circ}, D_{2}^{\circ}\right)$ is locally trivial over $T_{1}^{\circ}$. Moreover, a general fiber $F_{2}$ of $a_{2}^{\circ}:=a_{2 \mid X_{2}^{\circ}}$ is a smooth toric variety with boundary divisor $D_{2 \mid F_{2}}$. We may also assume without loss of generality that $X_{2}^{\circ}$ has complement of codimension at least 2 since there is no $a_{2}$-exceptional divisor on $X_{2}$.

Let $C_{2}$ be an irreducible component of $D_{2}$. The short exact sequence (see [KK08, Lemma 2.13.2])

$$
0 \rightarrow \mathscr{O}_{C_{2}} \rightarrow T_{X_{2}}\left(-\log D_{2}\right)_{\mid C_{2}} \rightarrow T_{C_{2}}\left(-\log \left(D_{2}-C_{2}\right)_{\mid C_{2}}\right) \rightarrow 0
$$

implies that $T_{C_{2}}\left(-\log \left(D_{2}-C_{2}\right)_{\mid C_{2}}\right)$ is R-flat. Moreover, $K_{C_{2}}+\left(D_{2}-C_{2}\right)_{\mid C_{2}} \sim_{\mathbb{Q}} 0$. The induction hypothesis applied to $C_{2}$ then says that there exists a smooth projective variety $T_{2}$ with $K_{T_{2}} \equiv 0$ as well as a smooth morphism with rational fibers $b_{2}: C_{2} \rightarrow T_{2}$. Moreover, the fibration $\left(C_{2},\left(D_{2}-C_{2}\right)_{\mid C_{2}}\right) \rightarrow T_{2}$ is locally trivial for the analytic topology and $T_{2}$ contains no rational curve.

Notice that fibers of $C_{2} \cap X_{2}^{\circ} \rightarrow T_{1}^{\circ}$ are projective with rational connected components. On the other hand, the number of connected components of $C_{2} \cap a_{2}^{-1}(t)$ does not depend on the point $t \in T_{1}^{\circ}$ since $\left(X_{2}^{\circ}, D_{2}^{\circ}\right) \rightarrow T_{1}^{\circ}$ is a locally trivial fibration. Let $T_{3}^{\circ} \rightarrow T_{1}^{\circ}$ be the corresponding étale cover, and let $T_{3}$ be the normalization of $T_{1}$ in the function field of $T_{1}^{\circ}$. Note that $T_{3} \rightarrow T_{1}$ is quasi-étale. In particular, $T_{3}$ is klt and $K_{T_{3}} \equiv 0$. Moreover, there is a birational map $\iota: T_{3} \rightarrow T_{2}$. Since $T_{2}$ contains no rational curve and $T_{3}$ is klt, $\iota$ is a morphism by [HM07, Corollary 1.7]. On the other hand, since $T_{2}$ is smooth and both $T_{2}$ and $T_{3}$ have numerically trivial canonical class, we conclude that $\iota$ is an isomorphism. In particular, $T_{3}$ is smooth and contains no rational curve.

Replacing $T_{1}$ by $T_{3}$ and $X_{1}$ by a quasi-étale cover, if necessary, we may assume without loss of generality that the following holds.

Assumption 6.3. The variety $T_{1}$ is smooth and contains no rational curve.

Step 4. Then [HM07, Corollary 1.7] implies that both $a_{1}$ and $a_{2}$ are morphisms. By the Nagata-Zariski purity theorem, there is an étale morphism $\eta: T_{2} \rightarrow T_{1}$ such that $\eta \circ b_{2}=a_{2 \mid C_{2}}$. Now, we have a commutative diagram as follows:

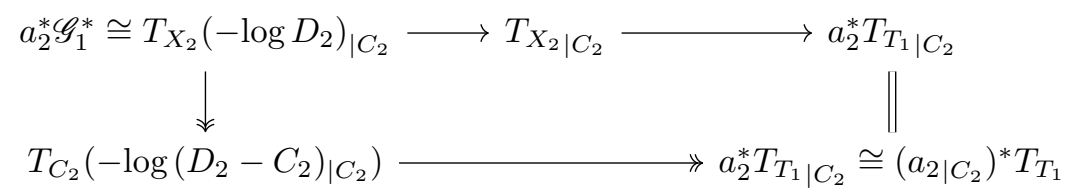

It follows that the map $\mathscr{G}_{1}^{*} \rightarrow T_{T_{1}}$ induced by the composed map

$$
a_{2}^{*} \mathscr{G}_{1}^{*} \cong T_{X_{2}}\left(-\log D_{2}\right) \rightarrow T_{X_{2}} \rightarrow a_{2}^{*} T_{T_{1}}
$$

is surjective. Arguing as above, this implies that the fibration $\left(X_{2}, D_{2}\right) \rightarrow T_{1}$ is locally trivial for the analytic topology. By Step 1, we see that the fibration $\left(X_{1}, D_{1}\right) \rightarrow T_{1}$ is also locally trivial.

Step 5. Let $f: X \rightarrow R$ be the maximally rationally chain connected fibration. Recall that $f$ is an almost proper map and that its general fibers are rationally connected. Let $\omega \in H^{0}\left(X, \Omega_{X}^{q} \otimes \mathscr{L}\right)$ be a twisted $q$-form defining the foliation $\mathscr{H}$ on $X$ induced by $f$. By Proposition 5.6, any irreducible component of $D$ maps onto $R$. This implies that the zero set of the reflexive pull-back $\omega_{1} \in H^{0}\left(X_{1}, \Omega_{X_{1}}^{[q]} \otimes \gamma^{*} \mathscr{L}\right)$ of $\omega$ has codimension at least 2 (see for instance [Dru18, Lemma 3.4]). Moreover, $\omega_{1}$ obviously defines the foliation $\mathscr{H}_{1}$ on $X_{1}$ induced by the map $a_{1}$. It follows that $\gamma^{*} \mathscr{L} \cong \operatorname{det} \mathscr{N}_{\mathscr{H}_{1}} \cong \mathscr{O}_{X_{1}}$. Let $\omega_{2} \in H^{0}\left(X_{2}, \Omega_{X_{2}}^{q}\right)$ be the pull-back of $\omega$ to $X_{2}$. Then $\omega_{2}$ defines the foliation $\mathscr{H}_{2}$ on $X_{2}$ induced by the map $a_{2}$. Since $\operatorname{det} \mathscr{N}_{\mathscr{H}_{2}} \cong \mathscr{O}_{X_{2}}$ and $\mathscr{H}_{2}$ is regular, we conclude that $\omega_{2}$ is nowhere vanishing. This in turn implies that $\mathscr{H}$ is regular.

By Lemma 6.4 below, $f$ extends to a smooth morphism with rationally connected fibers $a: X \rightarrow T$ onto a smooth projective variety $T$. By Theorem 1.9 , there is a locally free, R-flat sheaf $\mathscr{G}$ on $T$ such that $a^{*} \mathscr{G} \cong$ $\Omega_{X}^{1}(\log D)$. Arguing again as above, we conclude that the fibration $(X, D) \rightarrow T$ is locally trivial for the analytic topology and that any fiber $F$ of $a$ is a smooth toric variety with boundary divisor $D_{\mid F}$.

Notice that $K_{T}$ is torsion since $\gamma^{*} \mathscr{L} \cong \mathscr{O}_{X_{1}}$. In particular, $T$ is not uniruled. This easily implies that the image on $X$ of any fiber of $a_{1}$ is contracted by $a$. On the other hand, if $F$ is a general fiber of $a$, then any connected component of $(\gamma \circ \beta)^{-1}(F)$ is rationally connected. This immediately implies that $\operatorname{dim} T=\operatorname{dim} T_{1}$. Moreover, by the rigidity lemma, there is a finite morphism $\tau: T_{1} \rightarrow T$ such that $a \circ \gamma=\tau \circ a_{1}$. Since $T$ is 
smooth and both $T$ and $T_{1}$ have numerically trivial canonical class, we conclude that $\tau$ is étale. It follows that $T$ contains no rational curve. This completes the proof of the theorem.

The following result is an immediate consequence of [Hör07, Corollary 2.11].

Lemma 6.4. Let $X$ be a complex projective manifold, and let $f: X \rightarrow Y$ be an almost proper dominant rational map onto a normal projective variety $Y$. Suppose that the general fibers of $f$ are rationally connected. Suppose furthermore that the foliation $\mathscr{G}$ on $X$ induced by $f$ is regular. Then $f$ is a smooth morphism. In particular, $Y$ is smooth.

Proof of Corollary 1.5. By Theorem 1.1, there exist a smooth projective variety $T$ with $K_{T} \equiv 0$ as well as a smooth morphism with connected fibers $a: X \rightarrow T$. The fibration $(X, D) \rightarrow T$ is locally trivial for the analytic topology and any fiber $F$ of the map $a$ is a smooth toric variety with boundary divisor $D_{\mid F}$. Since $\pi_{1}(X)=\{1\}$ by assumption, we also have $\pi_{1}(T)=\{1\}$. It follows that $K_{T} \sim_{\mathbb{Z}} 0$. This in turn implies that $h^{p, 0}(X) \geqslant 1$ where $p:=\operatorname{dim} T$. Therefore, we must have $p=0$, proving the corollary.

Proof of Corollary 1.7. Notice that $T_{X}(-\log D)$ is $R$-flat. Moreover, $K_{X}+D \equiv 0$, so that Theorem 1.1 applies. There exist a smooth projective variety $T$ with $K_{T} \equiv 0$ as well as a smooth morphism with connected fibers $a: X \rightarrow T$. The fibration $(X, D) \rightarrow T$ is locally trivial for the analytic topology and any fiber $F$ of the map $a$ is a smooth toric variety with boundary divisor $D_{\mid F}$. By Theorem 1.9, there exists a vector bundle $\mathscr{G}$ on $T$ such that $a^{*} \mathscr{G} \cong T_{X}(-\log D)$. Set $p:=\operatorname{dim} T$. By Lemma 2.8, there is a nonzero map $\wedge^{p} \mathscr{G} \rightarrow \mathscr{O}_{T}\left(-K_{T}\right)$. Notice that both $\wedge^{p} \mathscr{G}$ and $\mathscr{O}_{T}\left(-K_{T}\right)$ are numerically flat. Thus, applying [DPS94, Proposition 1.16], we see that the morphism of locally free sheaves $\mathscr{G} \rightarrow T_{T}$ is surjective. This in turn implies that $T_{T}$ is numerically flat. By [DPS94, Corollary 1.19], we have $c_{1}(T)=0$ and $c_{2}(T)=0$. As a classical consequence of Yau's theorem on the existence of a Kähler-Einstein metric, $T$ is then covered by a complex torus (see [Kob87, Chapter IV Corollary 4.15]). This finishes the proof of the corollary.

Proof of Proposition 1.8. Note that all Chern classes of $T_{X}(-\log D)$ vanish. By [CP19, Theorem 1.3] (see also [Sch17, Theorem 4]), $T_{X}(-\log D)$ is slope-semistable with respect to any polarization. Then [Sim92, Corollary 3.10] implies that $T_{X}(-\log D)$ is numerically flat so that Corollary 1.7 applies, proving the corollary.

\section{REFERENCES}

[AD14] Carolina Araujo and Stéphane Druel, On codimension 1 del Pezzo foliations on varieties with mild singularities, Math. Ann. 360 (2014), no. 3-4, 769-798.

[Amb04] Florin Ambro, Shokurov's boundary property, J. Differential Geom. 67 (2004), no. 2, 229-255.

[BCHM10] Caucher Birkar, Paolo Cascini, Christopher D. Hacon, and James McKernan, Existence of minimal models for varieties of log general type, J. Amer. Math. Soc. 23 (2010), no. 2, 405-468.

[BdS09] Indranil Biswas and João Pedro P. dos Santos, On the vector bundles over rationally connected varieties, C. R. Math. Acad. Sci. Paris 347 (2009), no. 19-20, 1173-1176.

[Ber17] Hannah Bergner, On the lipman-zariski conjecture for logarithmic vector fields on log canonical pairs, preprint arXiv: 1712.04052, 2017.

[CKP12] Frédéric Campana, Vincent Koziarz, and Mihai Păun, Numerical character of the effectivity of adjoint line bundles, Ann. Inst. Fourier (Grenoble) 62 (2012), no. 1, 107-119.

[CP19] Frédéric Campana and Mihai Păun, Foliations with positive slopes and birational stability of orbifold cotangent bundles, Publ. Math. Inst. Hautes Études Sci. 129 (2019), 1-49.

[Del70] Pierre Deligne, équations différentielles à points singuliers réguliers, Lecture Notes in Mathematics, Vol. 163, SpringerVerlag, Berlin-New York, 1970.

[DPS94] Jean-Pierre Demailly, Thomas Peternell, and Michael Schneider, Compact complex manifolds with numerically effective tangent bundles, J. Algebraic Geom. 3 (1994), no. 2, 295-345.

[Dru14] Stéphane Druel, The Zariski-Lipman conjecture for log canonical spaces, Bull. Lond. Math. Soc. 46 (2014), no. 4, $827-835$.

[Dru18] Codimension one foliations with numerically trivial canonical class on singular spaces, preprint arXiv:1809.06905, 2018.

[Fuj99] Osamu Fujino, Applications of Kawamata's positivity theorem, Proc. Japan Acad. Ser. A Math. Sci. 75 (1999), no. 6, $75-79$.

[Fuj04] - Higher direct images of log canonical divisors, J. Differential Geom. 66 (2004), no. 3, 453-479.

[Fuj11] Non-vanishing theorem for log canonical pairs, J. Algebraic Geom. 20 (2011), no. 4, 771-783.

[Ful98] William Fulton, Intersection theory, second ed., Ergebnisse der Mathematik und ihrer Grenzgebiete. 3. Folge. A Series of Modern Surveys in Mathematics [Results in Mathematics and Related Areas. 3rd Series. A Series of Modern Surveys in Mathematics], vol. 2, Springer-Verlag, Berlin, 1998.

[GHS03] T. Graber, J. Harris, and J. Starr, Families of rationally connected varieties, J. Amer. Math. Soc. 16 (2003), no. 1, 57-67 (electronic). 
[GK14] Patrick Graf and Sándor J. Kovács, An optimal extension theorem for 1-forms and the Lipman-Zariski conjecture, Doc. Math. 19 (2014), 815-830.

[GKK10] Daniel Greb, Stefan Kebekus, and Sándor J. Kovács, Extension theorems for differential forms and Bogomolov-Sommese vanishing on log canonical varieties, Compos. Math. 146 (2010), no. 1, 193-219.

[GKPT19] Daniel Greb, Stefan Kebekus, Thomas Peternell, and Behrouz Taji, Nonabelian Hodge theory for klt spaces and descent theorems for vector bundles, Compos. Math. 155 (2019), no. 2, 289-323.

[Har80] Robin Hartshorne, Stable reflexive sheaves, Math. Ann. 254 (1980), no. 2, 121-176.

[HM07] Christopher D. Hacon and James Mckernan, On Shokurov's rational connectedness conjecture, Duke Math. J. 138 (2007), no. 1, 119-136.

[Hör07] Andreas Höring, Uniruled varieties with split tangent bundle, Math. Z. 256 (2007), no. 3, 465-479.

[KK08] Stefan Kebekus and Sándor J. Kovács, Families of canonically polarized varieties over surfaces, Invent. Math. 172 (2008), no. 3, 657-682.

[KM98] János Kollár and Shigefumi Mori, Birational geometry of algebraic varieties, Cambridge Tracts in Mathematics, vol. 134, Cambridge University Press, Cambridge, 1998, With the collaboration of C. H. Clemens and A. Corti, Translated from the 1998 Japanese original.

[KMM87] Yujiro Kawamata, Katsumi Matsuda, and Kenji Matsuki, Introduction to the minimal model problem, Algebraic geometry, Sendai, 1985, Adv. Stud. Pure Math., vol. 10, North-Holland, Amsterdam, 1987, pp. 283-360.

[Kob87] Shoshichi Kobayashi, Differential geometry of complex vector bundles, Publications of the Mathematical Society of Japan, vol. 15, Princeton University Press, Princeton, NJ; Princeton University Press, Princeton, NJ, 1987, Kanô Memorial Lectures, 5.

[Kol97] János Kollár, Singularities of pairs, Algebraic geometry—Santa Cruz 1995, Proc. Sympos. Pure Math., vol. 62, AMS, 1997, pp. 221-287.

[Kol07] János Kollár, Kodaira's canonical bundle formula and adjunction, Flips for 3-folds and 4-folds, Oxford Lecture Ser. Math. Appl., vol. 35, Oxford Univ. Press, Oxford, 2007, pp. 134-162.

[Kol13] János Kollár, Singularities of the minimal model program, Cambridge Tracts in Mathematics, vol. 200, Cambridge University Press, Cambridge, 2013, With a collaboration of Sándor Kovács.

[MM86] Yoichi Miyaoka and Shigefumi Mori, A numerical criterion for uniruledness, Ann. of Math. (2) 124 (1986), no. 1, 65-69.

[Sai80] Kyoji Saito, Theory of logarithmic differential forms and logarithmic vector fields, J. Fac. Sci. Univ. Tokyo Sect. IA Math. 27 (1980), no. 2, 265-291.

[Sch17] Christian Schnell, On a theorem of Campana and Păun, Épijournal Geom. Algébrique 1 (2017), Art. 8, 9.

[sga03] Revêtements étales et groupe fondamental (SGA 1), Documents Mathématiques (Paris) [Mathematical Documents (Paris)], vol. 3, Société Mathématique de France, Paris, 2003, Séminaire de géométrie algébrique du Bois Marie 196061. [Algebraic Geometry Seminar of Bois Marie 1960-61], Directed by A. Grothendieck, With two papers by M. Raynaud, Updated and annotated reprint of the 1971 original [Lecture Notes in Math., 224, Springer, Berlin].

[Sho92] V. V. Shokurov, Three-dimensional log perestroikas, Izv. Ross. Akad. Nauk Ser. Mat. 56 (1992), no. 1, $105-203$.

[Sim92] Carlos T. Simpson, Higgs bundles and local systems, Inst. Hautes Études Sci. Publ. Math. (1992), no. 75, 5-95.

[Vie95] Eckart Viehweg, Quasi-projective moduli for polarized manifolds, Ergebnisse der Mathematik und ihrer Grenzgebiete (3), vol. 30, Springer-Verlag, Berlin, 1995.

[Win04] Jörg Winkelmann, On manifolds with trivial logarithmic tangent bundle, Osaka J. Math. 41 (2004), no. 2, $473-484$.

[Zha05] Qi Zhang, On projective varieties with nef anticanonical divisors, Math. Ann. 332 (2005), no. 3, 697-703.

[Zha06] L Rational connectedness of log Q-Fano varieties, J. Reine Angew. Math. 590 (2006), 131-142.

Stéphane Druel: Univ Lyon, CNRS, Université Claude Bernard Lyon 1, UMR 5208, Institut Camille Jordan, F-69622 Villeurbanne, France

E-mail address: stephane.druel@math.cnrs.fr

Federico lo Bianco: Univ lyon, CNRs, Université Claude Bernard lyon 1, UMr 5208, Institut Camille Jordan, F-69622 Villeurbanne, France

E-mail address: lobianco@math.univ-lyon1.fr 